\title{
ReScribe: An Unrestrained Tool Combining Multiplex Recombineering and Minimal-PAM ScCas9 for Genome Recoding Pseudomonas putida
}

Enrique Asin-Garcia," Maria Martin-Pascual," Luis Garcia-Morales, Richard van Kranenburg, and Vitor A. P. Martins dos Santos*

Cite This: https://doi.org/10.1021/acssynbio.1c00297

Read Online

ABSTRACT: Genome recoding enables incorporating new functions into the DNA of microorganisms. By reassigning codons to noncanonical amino acids, the generation of new-to-nature proteins offers countless opportunities for bioproduction and biocontainment in industrial chassis. A key bottleneck in genome recoding efforts, however, is the low efficiency of recombineering, which hinders large-scale applications at acceptable speed and cost. To relieve this bottleneck, we developed ReScribe, a highly optimized recombineering tool enhanced by CRISPR-Cas9mediated counterselection built upon the minimal PAM 5'NNG-3' of the Streptococcus canis Cas9 (ScCas9). As a proof of concept, we used ReScribe to generate a minimally recoded strain of the industrial chassis Pseudomonas putida by replacing TAG stop codons (functioning as PAMs) of essential metabolic genes with the synonymous TAA. We showed that ReScribe enables nearly $100 \%$ engineering efficiency of multiple loci in P. putida, opening promising avenues for genome editing and applications thereof in this bacterium and beyond.

KEYWORDS: CRISPR-ScCas9-mediated counterselection, minimal-PAM, multiplexing, recoding, recombineering, Pseudomonas putida
$\mathrm{D}$ ue to its physiological robustness, stress resistance, metabolic versatility, and fast growth, Pseudomonas putida KT2440 has become a platform for metabolic engineering aimed at industrial and environmental applications. ${ }^{1-3}$ On top of its intrinsic features, the toolbox for genetic programming of this bacterium has significantly improved over the past years, propelling it to the front ranks of the synthetic biology platforms. ${ }^{4}$ However, its full potential is still held back by limitations on genome-scale editing that hinder ambitious bioengineering projects such as genome recoding. The ability to rewrite genomes provides the opportunity to incorporate new properties to the DNA of industrially relevant microorganisms, thereby increasing their value as biotechnological platforms. $^{5}$ By reassigning natural codons to noncanonical amino acids, this approach allows the synthesis of new-tofunction proteins and peptides, leading to a significantly expanded space for bespoke biocatalysis. ${ }^{6,7}$ Moreover, alterations in the translation machinery derived from the repurposing of codons can also be part of their biosafety assurance by impairing the ability of recoded microbes to express foreign DNA or by producing proteins that cannot be functionally expressed in other organisms. ${ }^{8,9}$ This impact on horizontal gene transfer and viral infection enhances the biosafety and stability of the engineered strains constituting a powerful biocontainment strategy. ${ }^{10}$

Over the past decade, a few recoding efforts have been published using different approaches. ${ }^{5,8,11-15}$ The first strategy used recombineering-based multiplex automated genome engineering (MAGE) to create a genome-wide recoded Escherichia coli. ${ }^{8}$ In that pioneering approach, all TAG stop codons were replaced with synonymous TAA codons, allowing the deletion of release factor 1 and the reassignment of UAG translation function to non-canonical amino acids (ncAA). ${ }^{16}$ This alteration allowed the incorporation of the recoded codon in essential genes conferring metabolic dependence on the ncAA for cell viability ${ }^{10,17}$ and hampering the dispersal of functional DNA from the synthetic chassis cell to natural microbes. ${ }^{15}$ Other strategies have been based on chemical synthesis of recoded DNA and its incorporation into the target

Received: June 29, 2021 
microbe either by substituting genome segments of different sizes ${ }^{5,13,14,18}$ or by substituting the entire genome. ${ }^{19}$ Despite their potential, these strategies keep requiring a costly DNA synthesis investment and a very laborious assembly process. Hence, there is a need for an efficient site-directed editing genome engineering tool to enable recoding.

Recombineering is a powerful genome editing technique based on a recombinase protein that promotes the incorporation of single-stranded DNA (ssDNA) molecules mimicking Okazaki fragments in the replication fork during DNA replication producing the intended mutation. ${ }^{20,21}$ Over the past years, different recombinases have been tested in $P$. putida including the Red $\beta$-recombinase and the RecET system from the E. coli Lambda phage and Rac prophage, the activity of which is relatively low in Pseudomonas species in absence of selection. ${ }^{4}$ Other $P$. putida-borne recombinases identified through genus-specific bioinformatic mining, Ssr and Rec2, have been experimentally validated with promising results. $^{22,23}$ Recently, Rec2-mediated ssDNA recombineering has been merged with transitional inhibition of the native mismatch machinery repair (MMR) system $^{24}$ by coexpressing a dominant-negative allele of $m u t L$, and further improved by iterating the recombineering protocol. Yet, efficiencies dropped dramatically when multiplexing, ${ }^{25}$ which prevents pursuing a full genome recoding and other high-throughput enterprises of mutations at genome-scale. The authors pointed to the core recombinase as the key limitation suggesting that a different or an optimized protein may work better in the proposed pipeline. Alternatives have already arisen in the way of (i) new recombinases like PapRecT, which has enabled efficient recombineering in the related species Pseudomonas aeruginosa; and (ii) optimization methods, such as RBS strengthening. ${ }^{26}$

In addition, CRISPR-Cas9 can be used as counterselection in recombineering by eliminating non-edited cells. To such end, the endonuclease Cas9, guided by the spacer, induces double-stranded DNA breaks (DSB) in the target site when the cell has not been mutated. In most bacteria, DSB can be prevented only via homology-directed repair (HDR) if a dsDNA template is provided. Therefore, in the absence of such template, cells will die as they typically lack a functional nonhomologous end joining (NHEJ) repair system. ${ }^{27}$ The targeting and cleavage specificity of Cas9 proteins requires two RNA elements, the precursor CRISPR RNA (crRNA), and the trans-activating RNA (tracrRNA). Each mature crRNA:tracrRNA:Cas9 ribonucleoprotein complex comprises a single transcribed spacer, a part of the neighboring repeat sequence, the tracrRNA and the Cas9. The design of the spacer allows directing the Cas9 protein to the desired protospacer (complementary spacer sequence present in the genome). The only requirement of the target site is the presence of a protospacer adjacent motif (PAM), a short (3-8 nucleotides) sequence, commonly found at the $3^{\prime}$ end of the protospacer that varies among Cas proteins.

CRISPR-Cas9-mediated counterselection has already been used for increasing the efficiency of recombineering in P. putida ${ }^{28}$ employing the paradigm Streptococcus pyogenes Cas9 (SpCas9), which needs the PAM sequence 5'-NGG-3'. This general version of the method presents limitations for high-throughput applications like bacterial genome recoding, in which the difference between the wild type and mutated genotypes can be a single nucleotide. First, this single change might be insufficient for preventing the Cas9 targeting when positioned in the protospacer sequence, since single mis- matches across the spacer sequence can be tolerated ${ }^{29}$ and recombinant cells would not be properly discriminated by the Cas9 cleavage. Furthermore, although short and abundant in high-GC content genomes such as the one of P. putida, the $5^{\prime}$ NGG-3' PAM is not available in every desired target site required for genome recoding. In this scenario, the deployment of SpCas9 as counterselection method becomes a serious challenge, if not an impossible enterprise.

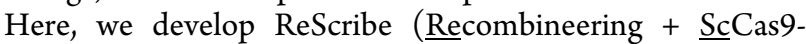
mediated counterselection), a highly efficient tool for genome recoding $P$. putida making use of the TAG stop codon itself as PAM for high on-target efficiencies. We boosted recombineering efficiencies by using the Streptococcus canis Cas9 ortholog, ScCas9. Previous in silico analysis showed a ScCas9 PAM specificity of $5^{\prime}$-NNGTT-3', which was later refined to $5^{\prime}$ NNG-3' in in vivo studies. ${ }^{30}$ Additionally, an engineered version of the ScCas9 in which the loop D367-376 had been removed showed a concomitant change in the specificity of the PAM from $5^{\prime}-\mathrm{NNG}-3^{\prime}$ to $5^{\prime}-\mathrm{NAG}-3^{\prime} .^{30}$ Both PAM sequences would allow to edit all possible TAG stop codons of the genome of $P$. putida KT2440, permitting an efficient counterselection after their recoding to TAA.

We thus propose ReScribe as a key solution to other multiple approaches that failed to surpass the reference recombineering efficiencies in $P$. putida KT2440 encompassing: (i) oligonucleotide design, (ii) RBS strengthening, and (iii) the alternative PapRecT recombinase. By using ReScribe, we showed near to $100 \%$ cleavage efficiencies with both ScCas9 and ScCas9 $\Delta$ loop using a wide range of spacers in different $P$. putida and $E$. coli strains, revealing their virtually unrestrained applicability in these two different bacteria. Moreover, ReScribe reached allelic replacement efficiencies higher than $90 \%$ after a single round of recombineering in single and multiple loci simultaneously. Ultimately, such impressive efficiencies allowed us to build a minimally recoded P. putida KT2440 strain, in which the TAG stop codons of essential metabolic genes were replaced by the synonymous TAA in a highly efficient manner. This first milestone evidences the power of our technology, not only as a mean for whole genome recoding, but also as an unprecedented tool for precise and specific targets, removing the PAM boundaries of other recombineering and CRISPR-Cas9-mediated counterselection methods.

\section{RESULTS}

Analysis of the $P$. putida Genome for Recoding. The $6.18 \mathrm{Mb}$ genome of $P$. putida KT2440 contains a total of 5671 open reading frames ${ }^{31}$ (NCBI accession number NC_002947.4). On the basis of this annotation and the aforementioned UAG recoding strategy (2011), ${ }^{16} 654$ genes that contain the least frequent TAG stop codon were computationally identified (Supplementary Table S6), representing $\sim 11.5 \%$ of the total, which is significantly higher than that of E. coli, probably related to the high GC-content of P. putida's genome. ${ }^{32,33}$ A comprehensive analysis of these genes displayed features such as genomic coordinates, orientation, and size. Moreover, other characteristics that might complicate codon conversion were considered, namely, overlapping reading frames and essentiality (Supplementary Table S6). The list included 67 genes in which the TAG stop codon overlaps a different reading frame. Out of these 67, in 35 instances the recoding of TAG to TAA would result in a nonsynonymous amino acid change in the product of the second 


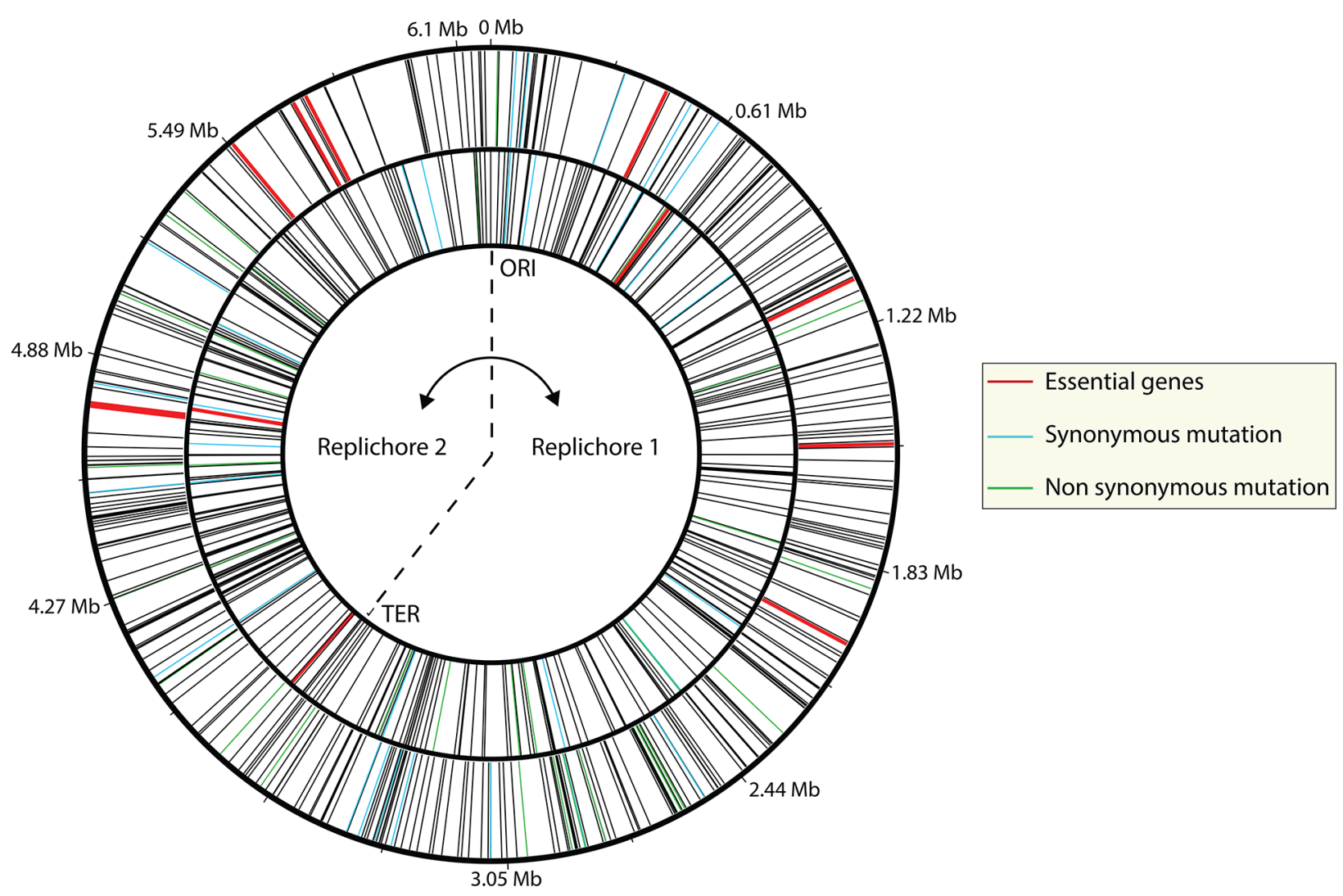

Figure 1. P. putida KT2440 genome's representation illustrating with lines the coordinates and orientation of all 654 genes terminated by TAG stop codons. TAG stop codons that are clockwise transcribed on the + DNA strand are depicted in the outer ring while those counterclockwise transcribed on the - DNA strand are represented in the inner ring. Essential genes terminated by TAG stop codons are indicated by the thick red lines. Genes finished in TAG stop codons whose mutation would result in a synonymous or non-synonymous amino acidic change on an overlapping gene are shown in blue or green, respectively. Genomic coordinates are represented around the circle, whereas origin of replication (ORI), terminus (TER), and replichores 1 and 2 are plotted in the inner circle.

reading frame, which could have an impact in the ultimate recoded phenotype and thus is not desired. Essentiality is a conditional feature subjected to the physiological context as a function of several factors. In absence of studies providing a comprehensive record of all essential genes of P. putida (e.g., high-density $\mathrm{TnSeq}^{34,35}$ or CRISPRi-based screening libraries), we defined the essential metabolic genes based on the predictions made through an experimentally validated genome-scale, constraint-based metabolic model. ${ }^{36-38}$ In order to identify the essential genes containing a TAG stop codon, a list of 270 conditionally essential genes in glucose minimal media was predicted using flux balance analysis (FBA) and the $P$. putida genome scale metabolic model iJP962. ${ }^{36}$ Of these, 12 were terminated by a TAG stop codon (Figure 1 and Supplementary Table S7).

Due to either poor efficiency or unsuitability of their selection methods, current genome editing tools did not seem suitable for conducting 654 mutations in P. putida. Therefore, for a task of this magnitude, it became imperative to develop a powerful new technique that relied on efficient multiplex recombineering.

RBS Optimization Strategy Does Not Increase ssDNA Recombineering Efficiency in $P$. putida. With an efficiency in the range of $10 \%$ of single target replacements after 10 iterative cycles, the recombineering protocol with recombinase $\operatorname{Rec} 2^{25}$ served as the baseline for the optimization study of our method. According to the results of our oligonucleotide optimization study (Figure 2), replacement efficiency was

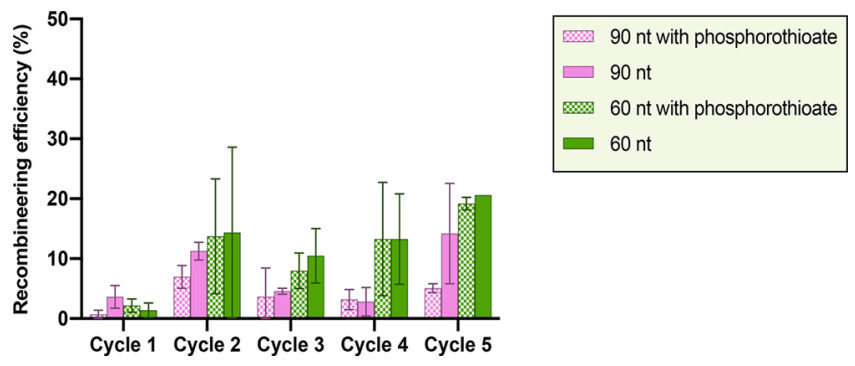

Figure 2. Assessment of oligonucleotide length and phosphorothioate modifications as oligonucleotide features that affect the allelic replacement frequency in P. putida KT2440. Evaluation is performed by screening of streptomycin resistant CFUs after mutation K43T of the $r p s L$ gene during 5 iterations of the recombineering cycle using Rec2. Recombineering efficiency was calculated in appropriate dilutions as the ratio between streptomycin resistant CFUs growing in LB-sm plates and total CFUs growing in LB plates. Replacement efficiency as a function of oligonucleotide length is depicted by colors, purple for 90 -mer and green for 60 -mer oligos, respectively. The effect in replacement efficiency of terminal phosphorothioate bonds positioned at both the $3^{\prime}$ and $5^{\prime}$ termini can be distinguished by dashed or full-colored bars, representing presence or absence of such backbone modifications, respectively. (Mean \pm s.d., $n=4$ biological).

highest when mediated by 60 -mer oligonucleotides. As a consequence, the oligos used in subsequent experiments were designed of 60 nucleotides in length, with the desired mutations included in the middle of the sequence. In addition, 
A

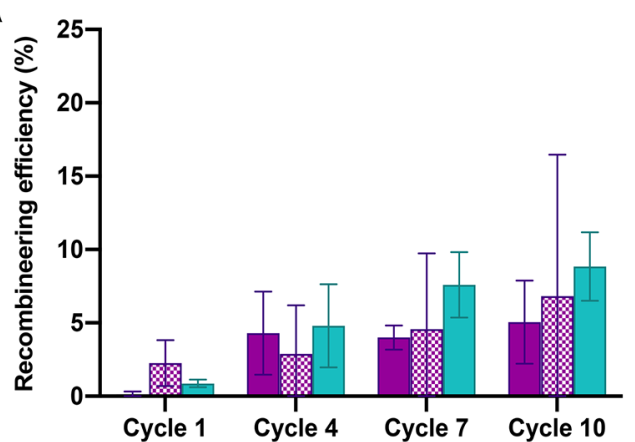

B

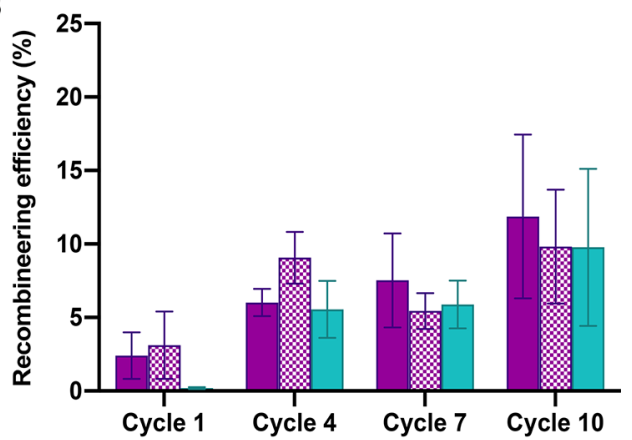

\begin{tabular}{|lllll}
\hline Rec2 & We Rec2 RBSopt $\quad \square$ PapRecT \\
\hline
\end{tabular}

C
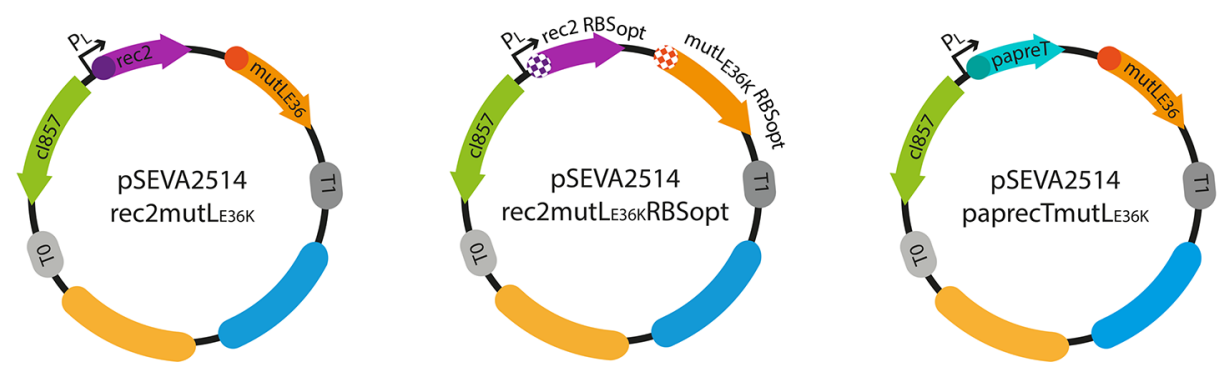

Figure 3. Comparison of allelic replacement efficiency of Rec2 with its RBS optimized version and with the alternative recombinase PapRecT. Recombineering was applied during 10 iterative cycles and samples were monitored after recovery steps of cycles 1, 4, 7, and 10. Graphs depict recombineering efficiency of Rec2, RBS optimized Rec2 and PapRecT mediating: (A) rpsL K43T mutation and (B) gfp stop66Y restoration mutation. Recombineering efficiency was calculated: (i) for the rpsL K43T mutation readout as the ratio between streptomycin resistant CFUs growing in LB-sm plates and total CFUs growing in LB plates; and (ii) for the gfp stop66Y restoration mutation as the ratio between fluorescent (mutated) and total CFUs. Noninduced recombinase samples were included as controls and were subtracted from the absolute values. (Mean \pm s.d., $n=4$ biological). (C) The pSEVA2514-rec2-mut $L_{\mathrm{E} 36 \mathrm{~K}}{ }_{\mathrm{PP}}$ plasmid, harboring the thermolabile cI857 repressor (green), the rec2 recombinase gene (purple) and $m u t L_{\mathrm{E} 36 \mathrm{~K}}{ }_{\mathrm{PP}}$ (orange). The pSEVA2514-rec2-mut $\mathrm{L}_{\mathrm{E} 36 \mathrm{~K}}{ }_{\mathrm{PBS}}{ }_{\mathrm{RBpt}}$ plasmid, harboring the thermolabile cI857 repressor (green), the RBS optimized rec2 recombinase gene (purple squared pattern) and the RBS optimized mutL $\mathrm{E}_{\mathrm{E} 6 \mathrm{~K}}{ }_{\mathrm{PP}}$ (orange squared pattern). The pSEVA2514paprecT-mut $L_{\mathrm{E} 36 \mathrm{~K}}{ }^{\mathrm{PP}}$ plasmid, harboring the thermolabile cI857 repressor (green), the paprecT recombinase gene (turquoise), and $\operatorname{mut}_{\mathrm{E} 36 \mathrm{~K}}{ }^{\mathrm{PP}}$ (orange).

while phosphorothioate bonds located at the terminal bases of recombineering oligonucleotides had been reported to increase the replacement efficiency in E. coli by evading nuclease degradation, ${ }^{39,40}$ our findings depicted in Figure 2 indicated that oligonucleotides with phosphorothioate bonds do not result in higher recombineering efficiency in $P$. putida. Therefore, phosphorothioate bonds were not included in the oligos used in this study.

In an attempt to enhance the editing efficiency of the reference plasmid pSEVA2514-rec2-mut $L_{\mathrm{E} 36 \mathrm{~K}}{ }^{\mathrm{PP}}$ by increasing the gene expression of its elements, the strongest predicted ribosomal binding sites (RBS) were designed and cloned upstream of both rec2 and $m u t L_{\mathrm{E} 36 \mathrm{~K}}{ }^{\mathrm{PP}}$ gene sequences. To evaluate the effect of the optimized variants, we used two different readouts to test the frequencies of mutant appearance: streptomycin resistance conferred by the K43T mutation in the rpsL gene in a WT P. putida KT2440 strain, and green fluorescence granted by the restoration of the genomic $g f p$ gene in $P$. putida Tn7GFPstop.

The results of applying 10 iterative recombineering cycles with oligonucleotides RO rpsL 60 and RO gfp stop, respectively, are depicted in Figure 3A and 3B. The percentage of mutated cells increased from $2.40 \%$ (Cycle 1) to $11.87 \%$ (Cycle 10) for $r p s L$, and from $0.13 \%$ (Cycle 1) to $5.05 \%$ (Cycle 10) for $g f p$, when using the pSEVA2514-rec2mut $_{\mathrm{E} 36 \mathrm{~K}}{ }^{\mathrm{PP}}$, which is in line with previously reported results. ${ }^{25}$
Yet, the RBS optimized variants (Figure 3C) carrying pSEVA2514-rec2 $2_{\mathrm{RBS} \text { opt }}-m u t L_{\mathrm{E} 36 \mathrm{~K}}{ }_{\mathrm{RB}}^{\mathrm{PP}}{ }_{\mathrm{RB}}$ opt did not render significant increases of editing efficiency nor did they seem to result in a burden for the cells. In this case, results indicated percentages in the range of $3.10 \%$ (Cycle 1) and $9.82 \%$ (Cycle 10) for $r p s L$, and $2.26 \%$ (Cycle 1) and $6.82 \%$ (Cycle 10) for $g f p$. Even though RBS optimization via RBS strengthening has been reported to increase recombineering levels with other recombinases, ${ }^{26}$ our results are in consistency with those previously obtained with Rec2 in the related species $P$. aeruginosa, ${ }^{26}$ concluding that most likely Rec2 activity is not limited by the levels of expression of the recombinase gene but by different intrinsic factors.

PapRecT as an Alternative to Rec2 for ssDNA Recombineering in P. putida. Next, our efforts focused on the replacement of Rec2. The nature of the core recombinase and its source have been shown to play an important role in the efficiency of recombineering ${ }^{42}$ suggesting that promising alternatives might be found among Pseudomonas species genomes and phages. Given its reported activity in other bacterial species, especially in the related $P$. aeruginosa, we selected PapRecT (originating from a $P$. aeruginosa phage $)^{26}$ for our setup in P. putida KT2440 (Figure 3C). The PapRecT recombinase resulted in recombineering efficiencies in the same range as those obtained with Rec2: $0.19 \%$ (Cycle 1) and 9.77\% (Cycle 10) for the rpsL readout; 
A
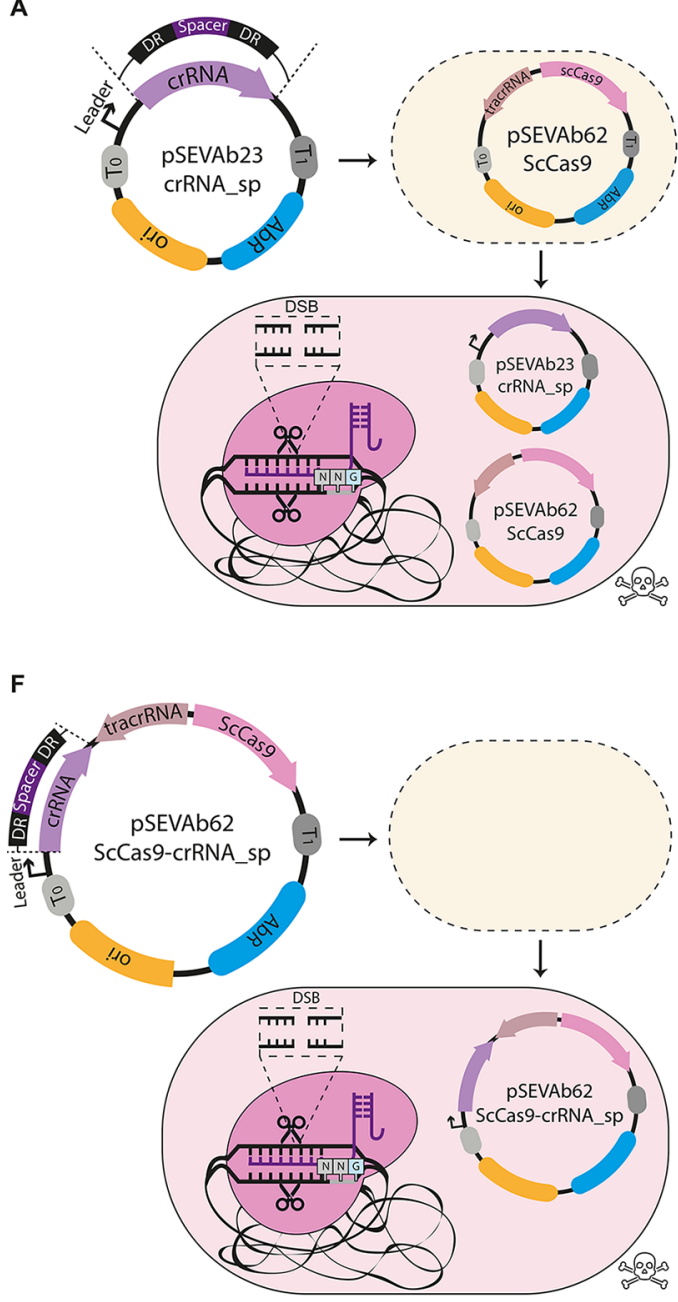

B

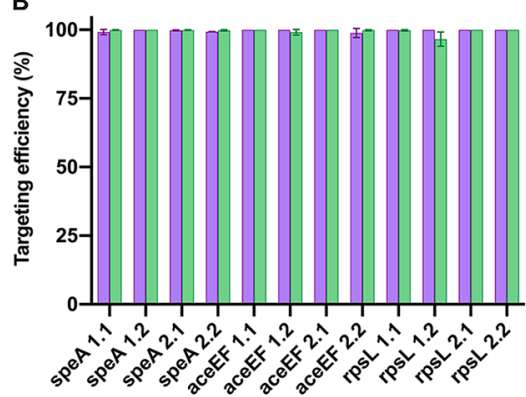

D

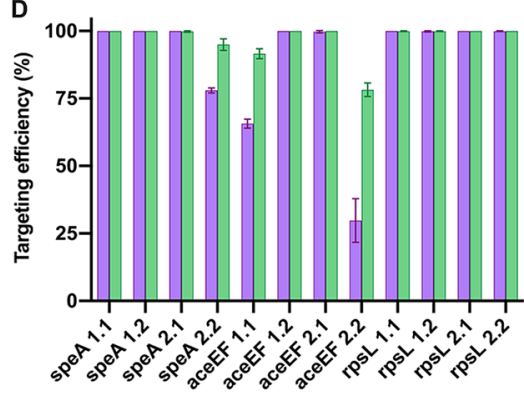

G

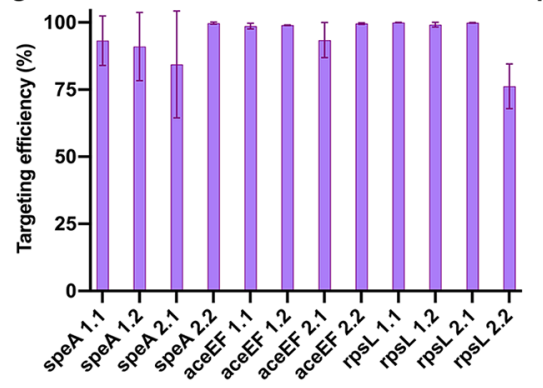

C

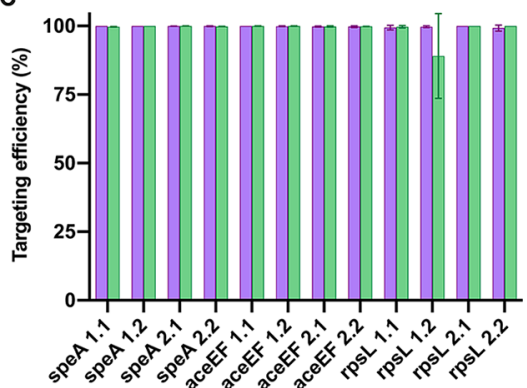

E

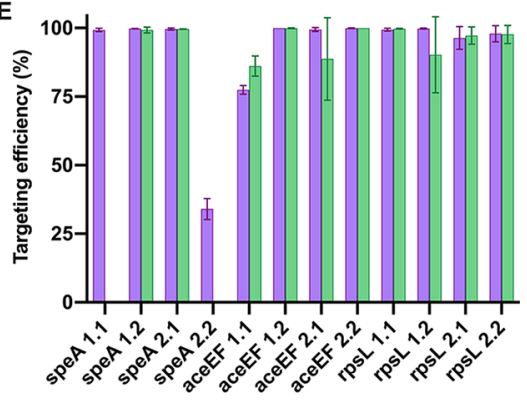

H

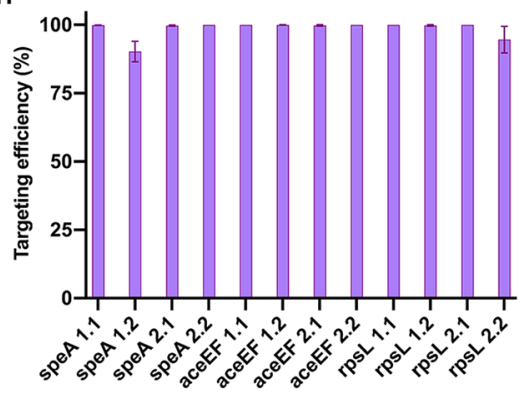

Figure 4. Cleavage assays with different ScCas9-based systems in P. putida and E. coli strains. (A) The two plasmids system is based on the pSEVAb23-crRNA and the pSEVAb62-ScCas9 plasmids. The pSEVAb23-crRNA plasmid harbors the crRNA comprised by the spacer (dark purple) interspersed by two direct repeats (black). The crRNA is expressed constitutively from the leader sequence. The pSEVAb23-crRNA plasmid is transformed into bacterial cells already harboring the pSEVAb62-ScCas9 plasmid. The pSEVAb62-ScCas9 plasmid expresses the ScCas9 (pink) and the tracrRNA (light brown) constitutively. The ScCas9:crRNA:tracrRNA complexes, directed by the spacer sequence, bind and unwind the target DNA, inducing a double strand break (DSB), causing bacterial cell death. The targeting efficiency is reported with different spacers targeting the genome of P. putida KT2440 (recA $A^{+}$(B), P. putida EM383 (recA $\left.A^{-}\right)(\mathrm{C})$, E. coli BL21 (rec $\left.A^{+}\right)(\mathrm{D})$, and E. coli DH5 $\alpha\left(r e c A^{-}\right)(\mathrm{E})$, expressing the ScCas9 (purple bars) or the ScCas9 $\Delta$ loop (green bars). (F) The one plasmid system is based on the pSEVAb62-ScCas-crRNA sp plasmid, which harbors the crRNA (light purple), the tracrRNA (light brown), and the ScCas9 (pink). All elements are expressed constitutively. After transformation the pSEVAb62-ScCas-crRNA_sp plasmid into bacterial cells, ScCas9:crRNA:tracrRNA complexes are formed, eliciting bacterial cell death. The targeting efficiency is reported with different spacers cleaving the genome of P. putida KT2440 (recA ${ }^{+}$) (G) and P. putida EM383 $\left(\mathrm{rec}^{-}\right)(\mathrm{H})$. The average targeting efficiency (\%) was calculated by normalizing the CFU numbers obtained with targeting spacers, with the CFU numbers obtained with nontargeting spacer (control) (mean \pm s.d., $n=3$ biological). Targeting spacers 1.1 have a PAM specificity of $5^{\prime}$ NBGTT-3', 1.2 of 5'-NBGVV-3', 2.1 of 5'-NAGTT-3' PAM, and 2.2 of 5'-NAGVV-3'.

and $0.87 \%$ (Cycle 1 ) and $8.85 \%$ (Cycle 10) for $g f p$ (Figure 3A and $3 \mathrm{~B})$. Although no significant differences could be seen between the results from both recombinases, PapRecT can be used as an equally valid alternative choice to the reference Rec2 in P. putida KT2440 due to their similar efficiency levels. In contrast to Rec2, the PapRecT RBS optimized version failed to provide any form of allelic replacement (data not shown).

ScCas9 Efficiently Cleaves $E$. coli and $P$. putida Genome. Unable to boost the recombineering efficiency by optimizing or replacing the core recombinase, we therefore aimed at developing a recombineering tool enhanced by CRISPR-Cas9-mediated counterselection that would reach the efficacy level required for multiscale engineering purposes. To this end, we needed a Cas protein with PAM specifications compatible with the TAG stop codon, which we found in the ScCas9 having $5^{\prime}$-NNG-3' as PAM.

First, we codon optimized the ScCas9 gene for P. putida KT2440 and analyzed its functionality by performing fluorescent loss assays (Supplementary Figure S1). We targeted different regions of a $s f g f p$ gene placed on a pSEVAb44 plasmid by using different spacers that were located next to a variety of $5^{\prime}$-NNG-3' PAMs. These preliminary results provided initial evidence that the codon optimized ScCas9 was functional in P. putida KT2440 and 


\section{A. Recombineering}

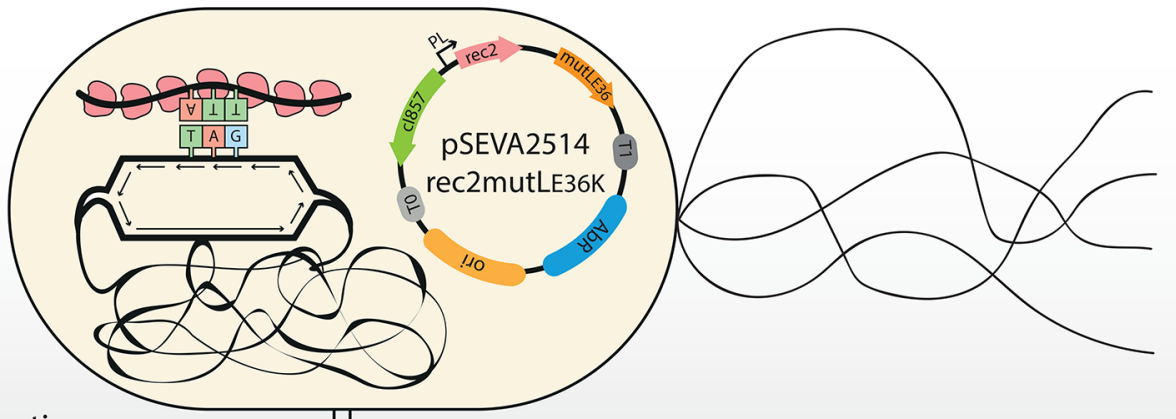

\section{B. ScCas9-mediated counterselection}

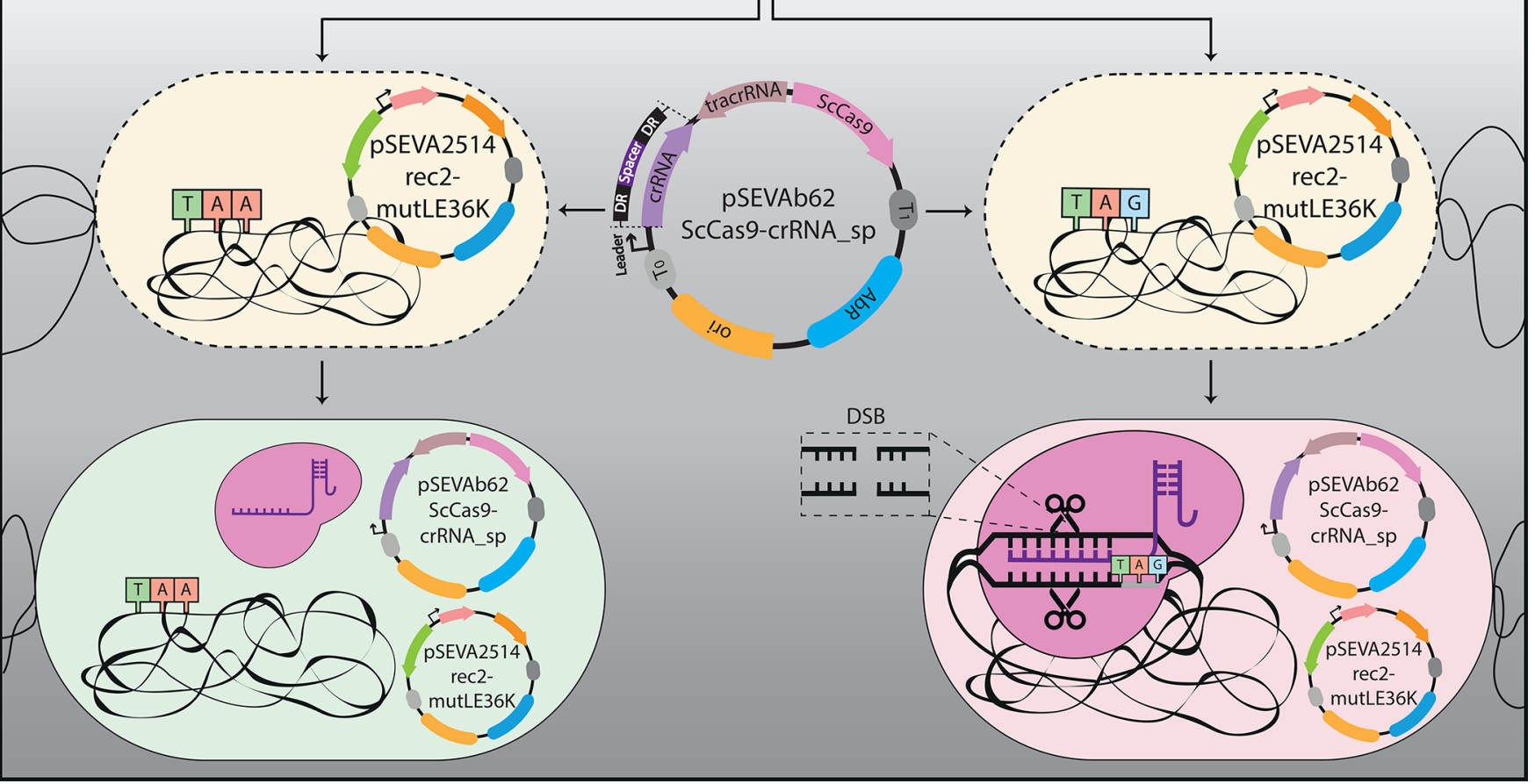

Figure 5. Enhanced genome-scale editing in P. putida with ReScribe. (A) The ssDNA oligonucleotide carrying TTA mutation and the pSEVA2514rec2-mut $L_{\mathrm{E} 36 \mathrm{~K}}{ }^{\mathrm{PP}}$ plasmid, harboring the thermolabile $c 1857$ repressor (green), the rec2 recombinase gene (light red), and $m u t L_{\mathrm{E} 36 \mathrm{~K}}{ }^{\mathrm{PP}}$ (light orange) are transformed to $P$. putida. The expression of both rec2 and $m u t L_{\mathrm{E} 36 \mathrm{~K}}{ }_{\mathrm{PP}}$ is controlled by the thermoinducible cI857/ $\mathrm{P}_{\mathrm{L}}$ system. With the increase of temperature from 30 to $42{ }^{\circ} \mathrm{C}$, the cI857 repressor is degraded and rec2 and mut $L_{\mathrm{E} 36 \mathrm{~K}}{ }^{\mathrm{PP}}$ are expressed. ${ }^{41}$ The expression of these two elements contributes to the incorporation of single nucleotide mutations in the genome of $P$. putida mediated via oligonucleotides that have been transformed. Consequently, a mix population of wild type and edited cells is generated. (B) The pSEVAb62-ScCas9-crRNA_sp plasmid, constitutively expressing all the CRISPR components (crRNA in light purple, tracrRNA in light brown and ScCas9 in pink), is transformed to the mixed population of $P$. putida wild type cells (genome with the TAG stop codon) and edited cells (genome with the TAA stop codon). The ScCas9:crRNA:tracrRNA ribonucleoprotein complex with a PAM specificity of 5'-NNG-3', recognizes the TAG as PAM and ScCas9 cleaves both strands. The double strand break (DSB) is lethal for P. putida wild type (cell with the light red background). In contrast, the edited cells have no PAM to be recognized in the site complementary to the spacer and escape ScCas9 activity (cell with light green background).

could target sequences adjacent to this minimal PAM. The spacer located next to a $5^{\prime}$-GAG-3' resulted in the most pronounced fluorescence loss, whereas the rest of the spacers showed different degrees of fluorescence depletion. While this preliminary experiment did not include a comprehensive number of spacers including all types of possible $5^{\prime}$-NNG-3' PAMs, it laid the groundwork for a more exhaustive analysis that included variations of a large array of factors such as characteristics of the minimal PAM, target genes, ScCas9 versions and bacterial systems. As very little was known about the use of this Cas9 variant with minimal PAM in bacteria, ${ }^{30}$ we included the bacterial model E. coli BL21 in our experiments. In addition, we also included P. putida EM383 and $E$. coli $\mathrm{DH} 5 \alpha$, both lacking the $\operatorname{rec} A$ gene, as a certain tolerance for weak spacers has been found in E. coli when the
HDR system is activated, ${ }^{43-45}$ with $r e c A$ being the main element of the HDR pathway.

We transformed a total of 12 pSEVAb23-crRNA_sp plasmids with different targeting spacers in $P$. putida KT2440, P. putida EM383, E. coli BL21 and E. coli DH5 $\alpha$, harboring the pSEVAb62-ScCas9 plasmid (Figure 4A) or the pSEVAb62-ScCas9 1 loop plasmid, in which the loop D367376 from the ScCas9 had been removed. We included both ScCas9 and ScCas9Dloop in our study since both their putative corresponding PAMs suit our purpose of using the TAG stop codon as PAM (5'-NNG-3' and $5^{\prime}$-NAG-3', respectively).

The 12 different spacers targeted 3 non-essential genes, $a c e E F, r p s L$, and speA to avoid that the absence of colonies was due to the interference of Cas9 instead of its cleavage 
A
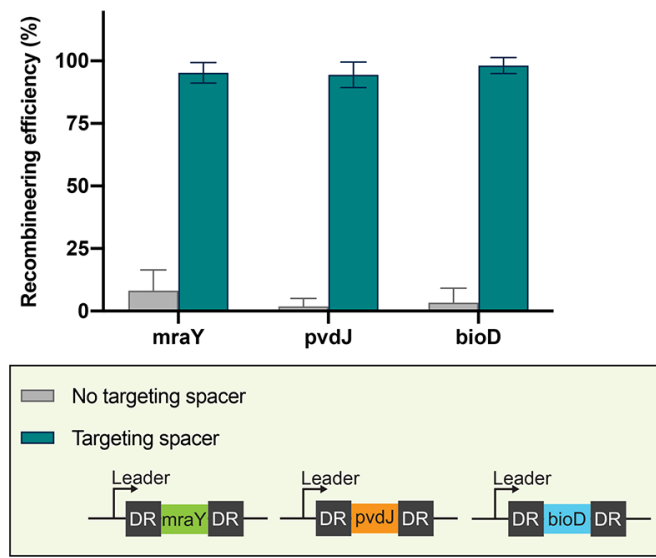

C

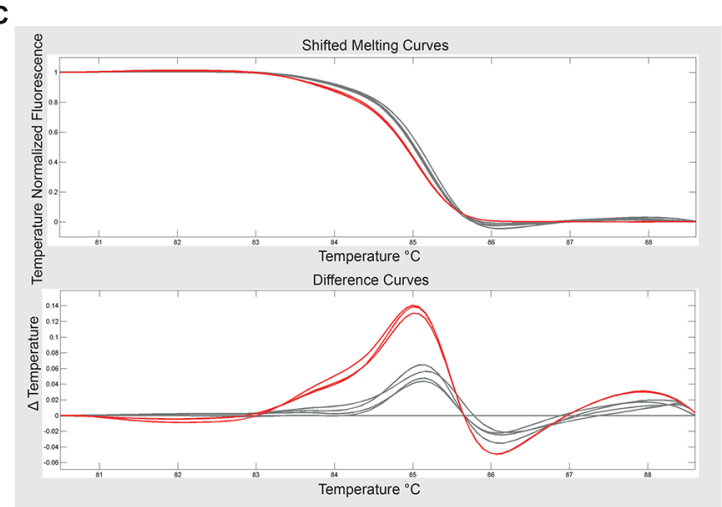

B
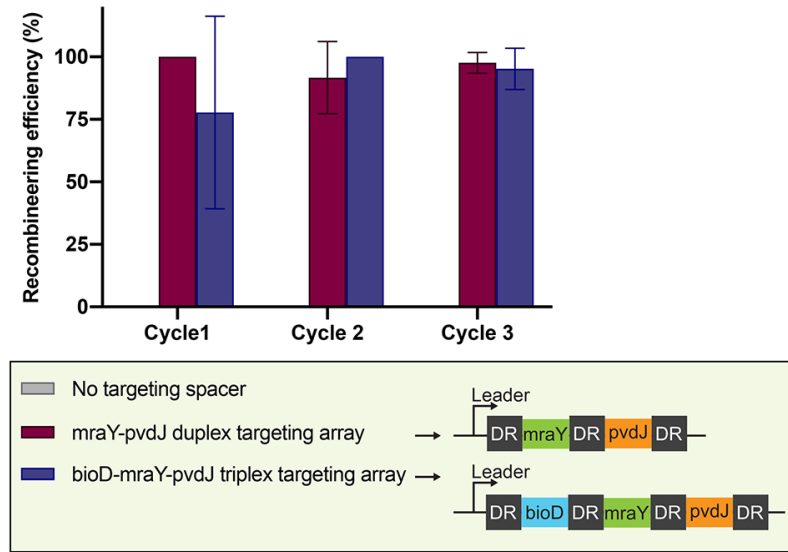

D

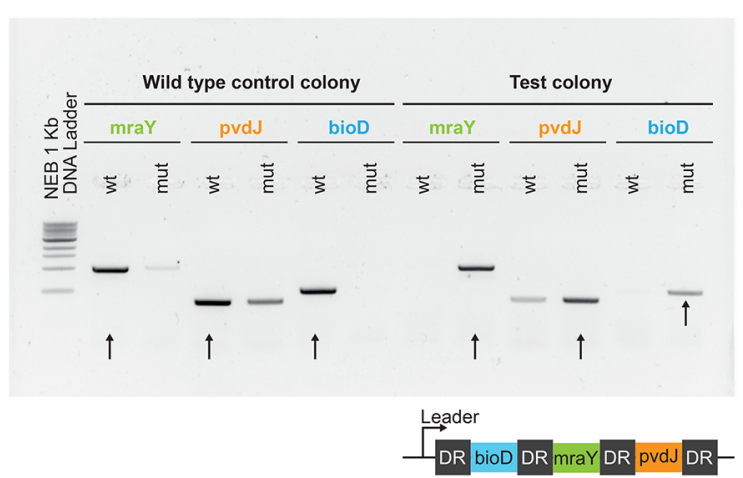

Figure 6. Allelic replacement efficiency for single and multiple targets with ReScribe in P. putida KT2440. (A) Recombineering efficiency of ReScribe for single targets. Recombineering was applied during 1 iterative cycle, after which the pSEVAb62-ScCas-crRNA_sp plasmid harboring different targeting spacers was electroporated for counterselection of wild type genotypes. The plasmid harboring a non-targeting spacer was used as control. Samples were monitored after recovery steps of cycle 1 and efficiency was calculated for samples with the non-targeting spacer (gray bars) and targeting spacer (turquoise bars) as the ratio between edited and nonedited colonies (mean \pm s.d., $n=3$ biological). (B) Recombineering efficiency of ReScribe for multiple targets. Recombineering was applied during 1, 2, and 3 iterative cycles, after which the pSEVAb62-ScCascrRNA_sp plasmid harboring duplex and triplex arrays was electroporated for counterselection of wild type genotypes. The plasmid harboring a non-targeting spacer was used as control. Samples were monitored after recovery steps of cycle 1, 2, and 3 and efficiency was calculated for samples with the non-targeting spacer (gray bars), mraY-pvdJ duplex array (dark red bars), and mraY-pvdJ-bioD triplex array (dark purple bars) as the ratio between edited and nonedited colonies (mean \pm s.d., $n \geq 2$ biological). (C) Single-nucleotide polymorphism TAG $\rightarrow$ TAA assessment by HRM. Interrogation by HRM analysis of the genotypes of a series of test colonies after mutation of mraY. HRM analysis is performed on PCR amplicons supplemented with a fluorescent dye by monitoring the separation of the two strands of DNA in real-time. Single-nucleotide mutations are observed as two different melt curves, wild type control curve (dark red) and test curve (gray), due to the high resolution of the process. (D) Single-nucleotide polymorphism TAG $\rightarrow$ TAA assessment by MASC-PCR. Comparison of MASC-PCR binary results between a wild type and a test colony with three targeted loci ( $m r a Y, p v d J$, bioD). Screening of each mutation is performed in two reactions: one with a FW $\mathrm{WT}_{\mathrm{W}}$ and RV pair of primers (wt) and another one with a $\mathrm{FW}_{\text {mut }}$ and $\mathrm{RV}$ pair (mut). $\mathrm{FW}_{\mathrm{WT}}$ and $\mathrm{FW}_{\text {mut }}$ primers are identical differing only in the $3^{\prime}$-terminal base which can be either a G or an A, consequently annealing to the WT or the mutant genotype, respectively. The wild type control colony showed stronger bands in the PCR reactions with the wt set of primers than in the PCR reactions with the mut set of primers, indicating that the genotype is wild type for mraY, pvdJ, and bioD genes. The test colony showed stronger bands in the PCR reactions with the mut set of primers than in the PCR reactions with the wt set of primers, indicating that the TAG stop codon of $m r a Y$, pvdJ, and bioD genes has been mutated to TAA.

activity. ${ }^{46,47}$ Additionally, the latter two loci had previously been targeted by SpCas9 in E. coli with high efficiency. ${ }^{43}$ For each locus, we designed 4 spacers with different PAMs that target different positions. The different PAMs: 5'-NBGTT-3', $5^{\prime}$-NBGVV-3', 5'NAGTT-3', and 5'NAGVV-3', were selected to analyze the PAM specificity of the ScCas9 and ScCas9 $\Delta$ loop and more specifically whether the nucleotides at positions 4 and 5 had higher specificity for $\mathrm{T}$ rather than $\mathrm{A}$, $\mathrm{G}$, and $\mathrm{C} .{ }^{30}$ We showed targeting efficiencies near to $100 \%$ with all the spacers in P. putida KT2440 (Figure 4B) and P. putida EM383 (Figure 4C) with both the ScCas9 and the ScCas9 $\Delta$ loop. Additionally, we also proved that these codonoptimized ScCas9 variants are highly efficient in E. coli BL21
(Figure 4D) and E. coli DH5 $\alpha$ (Figure 4E). In contrast to $P$. putida strains, in which all the spacers led to cell death at near $100 \%$ efficiency, E. coli strains survived with some spacers which were less efficient or had efficiencies similar to the nontargeting spacer.

In contrast to previous results in which the removal of the loop resulted in a concomitant change in the specificity of the PAM from the minimal $5^{\prime}-\mathrm{NNG}-3^{\prime}$ to $5^{\prime}-\mathrm{NAG}-3^{\prime},{ }^{30}$ here we show that ScCas9 $\Delta$ loop is equally able to cleave targets positioned next to the $5^{\prime}-\mathrm{NNG}-3^{\prime}$ PAMs as efficiently as the intact ScCas9 variant, both in P. putida and E. coli strains. Therefore, we decided to proceed with only the ScCas9 variant. With the perspective of combining both technologies: 
A

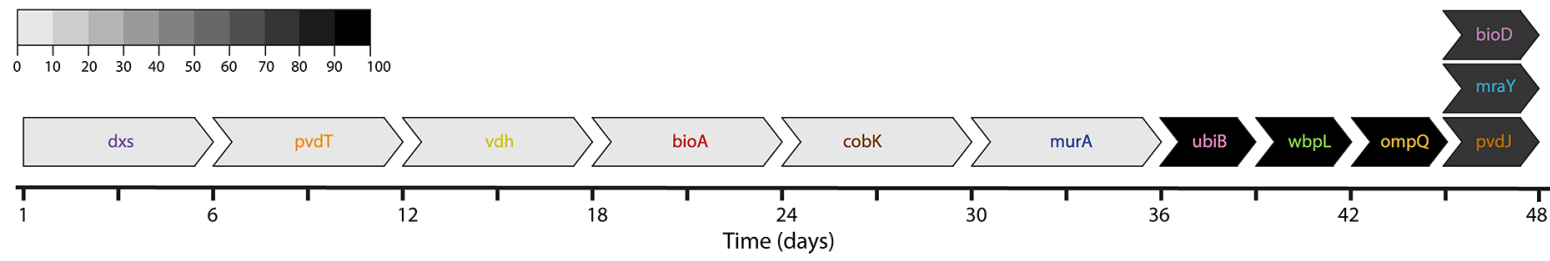

B
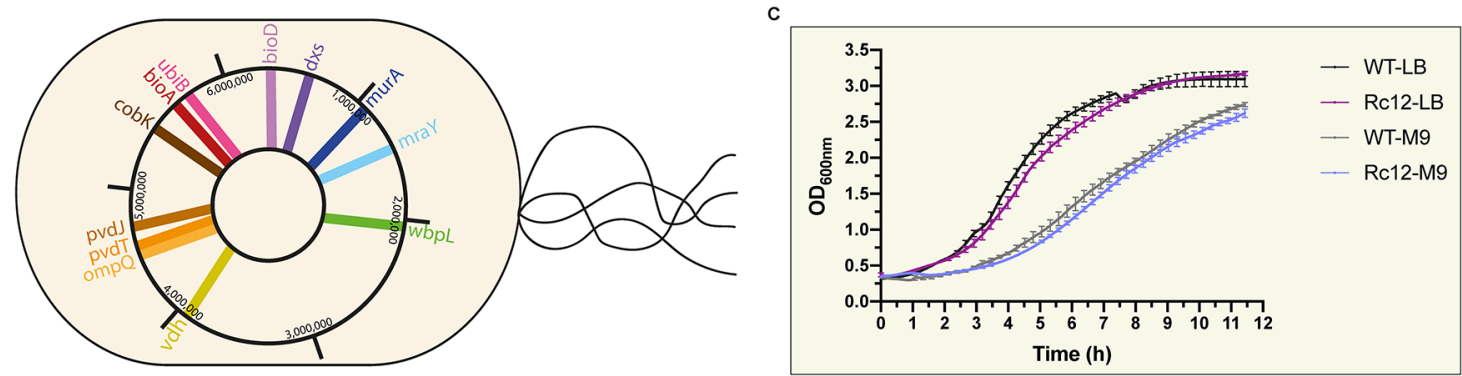

D
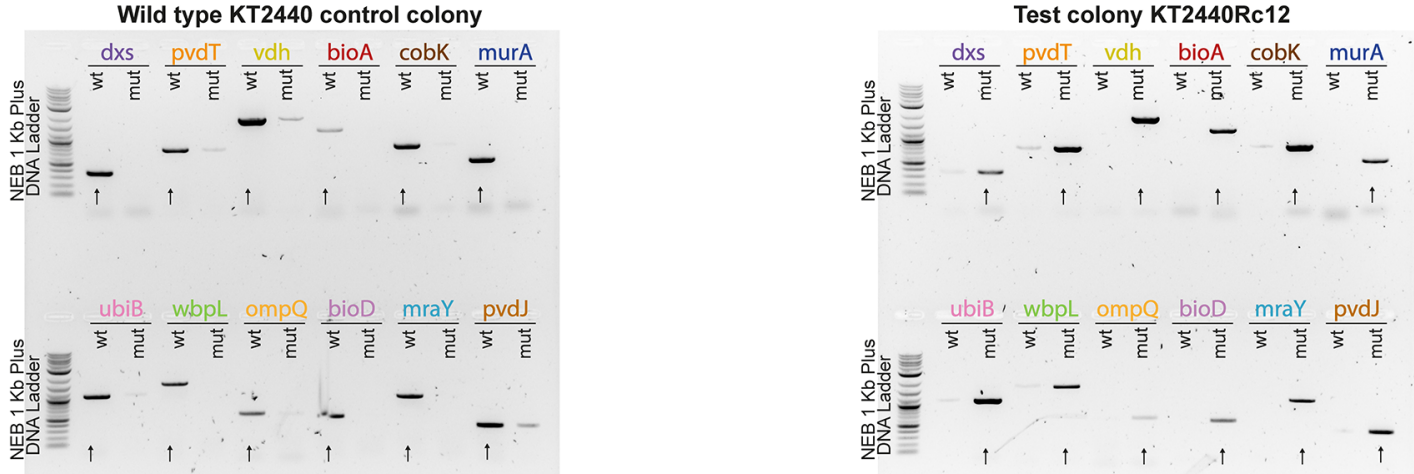

Figure 7. Minimally recoded $P$. putida KT2440Rc12 strain. (A) Timeline of the sequential mutation of the TAG stop codons in metabolic essential genes. Required time for performing each mutation is represented in days in the $\mathrm{X}$ axis while recombineering efficiency is depicted in percentage according to the gray scale. (B) Illustration of $P$. putida KT2440Rc12 genome portraying metabolic essential genes in glucose minimal medium terminated by TAG stop codons ( $d x s, p v d T$, $v d h$, bioA, cobK, murA, ubiB, wbpL, ompQ bioD, mraY, and $p v d J)$. (C) Fitness comparison between KT2440 (WT-LB) and KT2440Rc12 (Rc12-LB) in LB and M9-glucose media (WT-M9 and Rc12-M9). Data represent OD 600 over 12 h (mean \pm s.d., $n \geq 3$ biological replicates). (D) Comparison of MASC-PCR binary results between a wild type KT2440 control colony and a test colony KT2440Rc12. The wild type control colony showed stronger bands in the PCR reactions with the wt set of primers than in the PCR reactions with the mut set of primers, indicating that the genotype is wild type for all 12 genes. The test colony showed stronger bands in the PCR reactions with the mut set of primers than in the PCR reactions with the wt set of primers, indicating that the TAG stop codons of all 12 genes have been mutated to TAA.

CRISPR (ScCas9) and recombineering ( $\operatorname{Rec} 2)$, we aimed at simplifying the CRISPR-ScCas9 design by combining the 2 plasmids, the pSEVAb23-crRNA_sp and the pSEVAb62ScCas9, into one: the pSEVAb62-ScCas9-crRNA_sp plasmid. We cloned the same 12 spacers used in the 2-plasmid system into the pSEVAb62-ScCas9-crRNA_sp plasmid and transformed those plasmids in P. putida KT2440 and P. putida EM383 (Figure 4F). We showed efficiencies between $76 \%$ and $100 \%$ in P. putida KT2440 (Figure 4G) and $90-100 \%$ in P. putida EM383 (Figure 4H).

ScCas9 Counterselection Boosts the Efficiency of ssDNA Recombineering in $P$. putida. By converging recombineering and ScCas9-mediated counterselection we developed ReScribe, a method applying the unique features of ScCas9 that held the potential to enhance effectively the net efficiency of recombineering. In this study, Rec2 was maintained as core recombinase given its reported efficiency in a larger variety of loci. After one complete cycle of Rec2 recombineering (including recovery and segregation of the mutation), the heterogeneous cell population was subsequently transformed with a pSEVAb62-ScCas9-crRNA_sp targeting the wild type population, and therefore sifting for the engineered cells (Figure 5). To verify our hypothesis and in line with our initial recoding objective, we tested the unified ReScribe protocol with three essential genes of the genome of P. putida KT2440 that ended in TAG: mraY, pvdJ, and bioD. Individually, these three genes, were effectively mutated with efficiencies higher than $94 \%$ after only one recombineering iteration as demonstrated by MASC-PCR ${ }^{16,40}$ and $\mathrm{HRM}^{48,49}$ (Figure 6A, C, and D).

The high efficiency of the system for achieving individual single point mutations prompted us to test if ReScribe would enable the simultaneous mutagenesis of multiple loci, which in $P$. putida typically results in very low frequencies when relying on recombineering alone. ${ }^{25}$ Two or three oligonucleotides were cotransformed during the recombineering protocol and a single plasmid containing the ScCas9, tracrRNA, and a CRISPR array with the respective two or three spacers was used for selection. To increase our chances of generating a significant population of cells containing all combined 
mutations, counterselection was applied to different samples that had experienced 1,2 , and 3 cycles of recombineering. As a result of ScCas9's cleavage and the modest efficiencies of Rec2, wild type cells were wiped out from the population leading to plates with a significantly reduced number of colonies (Supplementary Table S8). This deficit was nonetheless outweighed by the high ratios of edited cells granted by ReScribe. After one single iteration, our selection system was already able to easily single out colonies containing two and three simultaneous mutations. Moreover, virtually every colony (97.6\% and $95.2 \%$ for two and three simultaneous mutations, respectively) presented all the intended alterations after three recombineering cycles (Figure 6B).

Though most cells without the desired mutations died from the DSB in the chromosome caused by the pSEVAb62ScCas9_crRNA_sp targeting plasmid, a small percentage was able to escape this lethal cleavage (Figure 5B). In addition, smaller colonies often appeared on selection plates upon prolonged incubation longer than the standard $24 \mathrm{~h}$, which turned-out to be false positives. These colonies are easily differentiated by visual inspection from the edited ones given their small size and late apparition.

To get a full understanding of these results, we further analyzed the aforementioned escapers by reculturing them on selection plates and sequencing all the CRISPR elements of the pSEVAb62-ScCas9_crRNA_mraY-pvdJ-(bioD) targeting plasmids. From the reculturing experiments, only few colonies were able to fully grow again in selection plates. Those were subsequently grown in liquid cultures and their plasmids were isolated and sequenced. Sequencing results showed miscellaneous cases of mutations and reorganizations, including recombination between the direct repeats of the CRISPR array or complete deletion of CRISPR-Cas9 machinery elements such as the tracrRNA, that would inactivate the pressure of the counterselection plasmid.

Recombineering vs ReScribe to Construct a Minimally Recoded $P$. putida Strain. Given the efficient and multisite recombineering possibilities granted by ReScribe, our next goal was to compare the standard Rec2-mediated recombineering and ReScribe in terms of efficiency and time, and to construct a minimally recoded $P$. putida strain. For such end, we used the informatic analysis previously described in this study and we aimed at recoding all predicted TAG codons (12 in total) that reside in conditionally essential genes in glucose minimal media.

As a proof of concept, we completed the recoding of all TAG codons by editing them to the synonymous TAA codon, generating the minimally recoded $P$. putida KT2440Rc12 strain by using both standard Rec2-mediated recombineering and ReScribe. Thereby, we highlighted the benefits of ReScribe in speed and efficiency when compared to the previous standard technique. The first six mutations of $P$. putida KT2440Rc12 (dxs, pvdT, vdh, bioA, cobK, murA) were introduced individually and consecutively via standard Rec2-mediated recombineering. This typically required 6 working days per mutation with an average efficiency of $8.3 \pm 2.8 \%$. Next, three mutations $(u b i B, w b p L$, ompQ) were performed making use of single-targeting ReScribe resulting in a decrease in working time from 6 to 3 days per mutation and a considerable increase of average efficiency to $90.5 \pm 9.9 \%$. Possibly due to a high plasmid burden imposed by the different targeting pSEVAb62ScCas9_crRNA_ (spacer) plasmids, easy isolation of plasmidcured colonies with an efficiency of $100 \%$ within $24 \mathrm{~h}$ was possible after two rounds of antibiotic-pressure-free media passaging. Finally, multiplex ReScribe was utilized for the simultaneous recoding of the three remaining genes (bioD, $m r a Y, p v d J)$ which was achieved in 3 days with an efficiency of $77.8 \pm 38.5 \%$ (Figure 7A). In comparison, ReScribe reduced the working time of standard Rec2-mediated recombineering to the half in its single-targeting version, and 6-fold when multiplexing, while it increased $\sim 10$-fold the efficiency levels.

Once completed (Figure 7B), the minimally recoded strain showed an unaffected fitness with equal doubling time to its ancestor, both in LB and M9-glucose (Figure 7C). Beyond the rapid screening performed with MASC-PCR (Figure 7D) and HRM, whole-genome sequencing confirmed the presence of all 12 mutations in the final KT2440Rc12 strain (Supplementary Table S9). This analysis revealed as well the presence of 40 offtarget mutations when compared with the reference $P$. putida KT2440 genome (accession no GCF_000007565.2) and the sequenced genome of an in-house reference strain from our laboratory (Supplementary Table S9). Considering that KT2440Rc12 had undergone at least 34 recombineering cycles (6 mutations with 5 cycles of standard ssDNA recombineering +3 mutations with 1 cycle of single ReScribe +3 mutations with 1 cycles of multiplex ReScribe; Figure 7A), the average number of off-target mutations would be 1.17 per recombineering cycle.

\section{DISCUSSION}

We developed ReScribe as a highly efficient method for multiplex recombineering of $P$. putida. The key element of ReScribe is the deployment of the minimal-PAM CRISPRScCas9 system, which provides single base-pair resolution and therefore permits the counterselection against wild type genotypes after introducing single-nucleotide polymorphisms. Taking advantage of the here validated minimal PAM 5'-NNG$3^{\prime}$, very precise and specific loci can be targeted in a highly efficient manner, which is otherwise impossible with CRISPR systems with more restricting PAMs. By applying ReScribe, we edited the genome of $P$. putida by substituting native TAG stop codons with the synonymous TAA stop codon with unprecedented efficiencies, 90-100\%, for both single and multiplex genome engineering. As a result, we built a minimally recoded $P$. putida KT2440 strain of essential metabolic genes, establishing the first step toward a whole-genome recoding process. The need for ReScribe was the result of failed attempts to increase the recombineering efficiency of the current reference Rec2 recombinase in $P$. putida $(10 \%$ efficiency for single targets and $2 \times 10^{-4} \%$ and $6 \times 10^{-6} \%$ efficiency for four and five targets, after 10 recombineering cycles). ${ }^{25}$ In this study, different factors were tested: (i) strandedness, structure, length, and backbone modifications of the recombineering oligonucleotides (Figure 2), (ii) expression of the Rec2 recombinase with strong synthetic RBSs (Figure 3), and (iii) PapRecT as an alternative recombinase to the baseline Rec2 (Figure 3). However, none of those changes led to significantly increased recombineering efficiencies, which are imperative for genome-scale applications such as our intended genome recoding.

The power of recombineering relies on the ability to rapidly edit the genome of organisms with high accuracy on a scale that is not feasible with previous traditional tools. For such an end, exceptionally high editing levels are crucial. ${ }^{50}$ Our findings demonstrate the great utility of the codon optimized ScCas9 variant to boost recombineering efficiencies in our system, 
which could be potentially reproduced in bacteria beyond P. putida. What makes ScCas9 and ReScribe especial, as compared to other CRISPR-Cas9-mediated counterselection systems, is the minimal PAM $5^{\prime}-\mathrm{NNG}-3^{\prime}$. It is worth mentioning that the ScCas9 $\Delta$ loop variant tested in this study was equally able to cleave targets adjacent to $5^{\prime}-\mathrm{NNG}-3^{\prime}$ PAMs despite previous in silico predictions that assigned it the more restricted 5'-NAG-3' PAM specificity. ${ }^{30}$ However, these results align with the in vitro assessment of the authors in human cells in which $\operatorname{ScCas} 9 \Delta$ loop was able to efficiently cleave at $5^{\prime}$ NGG-3' ${ }^{\prime}, 5^{\prime}$-NNGA-3' and $5^{\prime}$-NNGN-3' targets. ${ }^{30}$ While in this study the two variants were tested due to the suitability of both $5^{\prime}-\mathrm{NAG}-3^{\prime}$ and $5^{\prime}$-NNG-3' PAMs for the aimed objective, the latter PAM represents the most convenient choice for a broader applicability of the tool. This less restrained requirement expands dramatically the number of targetable sites and becomes critical when there is no flexibility in selecting protospacer sequences (which must be followed by the PAM sequence). ReScribe therefore represents an auspicious opportunity for those bacteria that, like P. putida, show limited recombineering activity with the currently available recombinases. ${ }^{51-55}$ Moreover, the potential niches of application of ReScribe go even further: given the high efficiency of the ScCas9 cleavage, the tool could be deployed for recalcitrant targets ${ }^{56,57}$ or for facilitating the process in bacteria with fully established recombineering systems such as E. coli. On top of that, ReScribe has demonstrated the feasibility of efficient multisite genome editing, which, in turn, enables further genome-scale engineering applications.

The major drawbacks of ReScribe are intrinsically connected to those of the parental techniques: Rec2-mediated recombineering and CRISPR-Cas9 technologies. In the first place, the efficiency of recombineering varies with the relative location of the gene. In this context, cold and hotspots for recombineering have been identified in other studies. ${ }^{64}$ Additionally, the position of the target loci with respect to the two replichores may have an effect too. Thus, genes closer to the origin of replication will be edited at higher levels than those located farther away. ${ }^{58,59}$ Moreover, the recombineering efficiency can also be affected by the nucleotide composition of the mutagenic oligonucleotide. Besides, in our particular case, the specific efficacy of Rec2 might be an extra limitation for the recombineering part of the workflow, especially for multiplexing experiments. Nonetheless, if any better alternative should exist, the core recombinase could easily be replaced, either for a better performance in P. putida or for the application of the pipeline in other organisms. The rapid emergence of high-throughput methods for surveying complex libraries of recombinases ${ }^{26}$ holds the potential of finding more suitable candidates, especially among Pseudomonas-borne counterparts for this particular case. At this point, account has to be taken of the intrinsic limitations of P. putida KT2440 as a receptor of exogenous DNA. While a better recombinase could improve the overall efficiency of the protocol, the poor ability of this bacterium to capture synthetic ssDNA could be an even greater constraint for recombineering in this species. ${ }^{25}$ Besides recombinase expression levels, recent studies highlighted additional interactions with single-stranded DNAbinding proteins (SSBs) within the replication fork as a way to improve recombineering efficiency in a given host. ${ }^{60,61} \mathrm{On}$ the basis of results reported in other Pseudomonas species, coexpression of these SSBs together with recombinases might be an option for enhancing the allelic replacement efficiencies even further. $^{62}$

Ultimately, increasing the efficiency of recombineering would be desired, not only as a way for eliminating the need of counterselection, but also for exploring higher-order selections than those showed in this work. Considering the number of required mutations, these orders of multiplexing (i.e., penta- to decaplex) would become useful for genomescale editing applications. While coexpression of a high number of crRNAs might be a challenge, it would not be impossible from multiple expression units. However, the generation of a cell population containing all the mutations, high enough to be set apart after the counterselection step, remains the most important obstacle, given the reported number of colonies that were obtained in our experiments with triplex selection.

Furthermore, ReScribe still has some of the limitations of the CRISPR technology, including (i) the need for constructing CRISPR plasmids directed at each modification locus; (ii) variations in the efficiency of the spacer, probably caused by differences in the secondary structure of the crRNA, which depends on the nucleotide composition of the spacer; ${ }^{63}$ and (iii) the loss of functionality of the CRISPR plasmid caused by homologous recombination between the direct repeats or by mutating one of the CRISPR elements. ${ }^{28,29,64}$ Regarding possible substitutions in the CRISPR-Cas 9 counterpart, the recently engineered $\mathrm{Sc}^{2+}$ and $\mathrm{HiFi}-\mathrm{Sc}^{2+}$ optimized ScCas9 variants could enhance the performance and robustness of ReScribe. ${ }^{65}$

Lastly, the significant number of 40 off-target mutations was found in the genome sequence of KT2440Rc12. While the overall count could be considered high, it is important to have into account the elevated number of recombineering cycles to which this strain was subjected. If we would consider offtargets per cycle, the average number of instances would be 1.17 , which is only slightly higher than recent reports about E. coli cells expressing a $\operatorname{Red} \beta$-recombineering system $(1.0 \pm$ 0.7 off-targets per recombineering cycle), and significantly lower than E. coli cells expressing the recombinase PapRecT $(3.3 \pm 0.6) .{ }^{26}$ While the accumulation of off-target mutations remains one of the main limitations of highly efficient recombineering systems, ${ }^{16,66}$ the number of off-targets observed in this study aligns with those obtained by other systems with considered low off-target mutagenesis, such as pORTMAGE2, 3, or $4 .^{24,66}$ Nevertheless, this negative effect deserves further investigation and needs to be dealt with special prudence when applying ReScribe to large-scale applications such as genome recoding.

In the context of recoding, the aforementioned limitations could be eventually overcome by following a strategy of highfidelity, total genomic synthesis. Contemporary DNA synthesis and assembly methods have enabled the generation of entire or largely synthetic genomes of Mycoplasma genitalium and Mycoplasma mycoides, ${ }^{67,68}$ Saccharomyces cerevisiae, ${ }^{12,69}$ and E. coli. ${ }^{14}$ Although those works provide a blueprint for future efforts, costs of whole-genome synthesis remain prohibitively expensive, accuracy of such lengthy sequences keep not being guaranteed, and assembly methods are still limited or insufficient in some particular organisms, thus hindering such approaches in most recoding undertakings. ${ }^{15}$

The tolerated stop codon changes already performed in the strain for essential genes support the feasibility of a wholegenome recoded project in P. putida. With ReScribe, this herculean enterprise could be carried out in separate strains in 
order to accelerate the process. Each strain can be used to recode a section of the genome (e.g., $100 \mathrm{~kb})$ which can then be assembled together in a single chromosome. For this convergence, the large edited genomic fragments can be captured in BAC (or YAC) plasmids and transformed into the recipient cell to replace the corresponding nonedited fragment using a CRISPR/Cas9-based strategy ${ }^{14}$ or a recombinasemediated cassette exchange. ${ }^{70}$

Overall, fully recoding $P$. putida would be a major step toward a new chemical landscape by enabling it to maximize and expand its attractive metabolic possibilities for bioproduction. The ability of reprogramming codons to encode alternative amino acids will allow the exploration of neotransmetabolisms, with the incorporation of elements and biochemistries beyond the cell's customary repertoire, e.g., silicon or halogens such as fluorine. ${ }^{7,71}$ These ncAA confer at the same time the opportunity of implementing a powerful genetic safeguard addressing both (i) biological isolation in defined environments with a supply of the ncAA, and (ii) genetic isolation by preventing horizontal gene transfer (HGT) of the neo-transgenes between organisms and across species. Such biosafety credentials would contribute to expand the possibilities for risk management of the strain and therefore could serve as a prelude for a more suitable and realistic consideration of $P$. putida for noncontained environmental applications. $^{72}$

While this work was conceived as a means of developing an efficient tool for recoding $P$. putida, we just had a glimpse of the capabilities of ReScribe. Our results support the hypothesis that counterselection can enhance the efficiencies of recombineering to nearly absolute levels in a multiplex manner and in an increased targetable space. ReScribe is therefore not limited by the size of the edit, the necessity of targeting gene by gene, or the location of a complex PAM. In addition, the proposed pipeline is neither restricted to $P$. putida nor hampered by the native mismatch repair machinery, which makes it a conveniently unrestrained tool for highly efficient engineering of arduous targets and endeavors.

\section{METHODS}

Bacterial Strains and Media. All bacterial strains with their respective characteristics used in the present study are listed in Supplementary Table S1. E. coli DH5 $\alpha$ and BL21 cells were made chemically competent as previously described. ${ }^{73}$ While the first were used for cloning purposes, fluorescence loss assays, and cleavage assays, the latter were only utilized for cleavage assays. Subsequently, electrocompetent $P$. putida strains were prepared as previously described ${ }^{74}$ and used for cleavage assays and recombineering experiments. Unless otherwise stated, $P$. putida and E. coli were cultured on LB (10 g/L NaCl, $10 \mathrm{~g} / \mathrm{L}$ tryptone, and $5 \mathrm{~g} / \mathrm{L}$ yeast extract) medium at 30 and $37^{\circ} \mathrm{C}$, respectively. Antibiotics were added when required, at the following concentrations: kanamycin, 50 $\mathrm{mg} / \mathrm{L}$; gentamicin, $10 \mathrm{mg} / \mathrm{L}$; chloramphenicol, $20 \mathrm{mg} / \mathrm{L}$; streptomycin, $50 \mathrm{mg} / \mathrm{L}$ and $100 \mathrm{mg} / \mathrm{L}$ for E. coli and P. putida, respectively. Fluorescence loss assays were performed on M9 minimal medium $\left(1.63 \mathrm{~g} / \mathrm{L} \mathrm{NaH}_{2} \mathrm{PO}_{4}, 3.88 \mathrm{~g} / \mathrm{L} \mathrm{K}_{2} \mathrm{HPO}_{4}, 2 \mathrm{~g} /\right.$ $\mathrm{L}\left(\mathrm{NH}_{4}\right)_{2} \mathrm{SO}_{4}, 10 \mathrm{mg} / \mathrm{L}$ EDTA, $100 \mathrm{mg} / \mathrm{L} \mathrm{MgCl}{ }_{2} \cdot 6 \mathrm{H}_{2} \mathrm{O}, 2 \mathrm{mg} /$ $\mathrm{L} \mathrm{ZnSO}_{4} \cdot 7 \mathrm{H}_{2} \mathrm{O}, 1 \mathrm{mg} / \mathrm{L} \mathrm{CaCl} \cdot 2 \mathrm{H}_{2} \mathrm{O}, 5 \mathrm{mg} / \mathrm{L} \mathrm{FeSO} \cdot \cdot 7 \mathrm{H}_{2} \mathrm{O}$, $0.2 \mathrm{mg} / \mathrm{L} \mathrm{Na}_{2} \mathrm{MoO}_{4} \cdot 2 \mathrm{H}_{2} \mathrm{O}, 0.2 \mathrm{mg} / \mathrm{L} \mathrm{CuSO} \cdot 5 \mathrm{H}_{2} \mathrm{O}, 0.4 \mathrm{mg} / \mathrm{L}$ $\mathrm{CoCl}_{2} \cdot 6 \mathrm{H}_{2} \mathrm{O}$, and $\left.1 \mathrm{mg} / \mathrm{L} \mathrm{MnCl}_{2} \cdot 2 \mathrm{H}_{2} \mathrm{O}\right)$ supplemented with $70 \mathrm{mM}$ of glucose. Recombineering experiments were performed on TB medium (12 g/L tryptone, $24 \mathrm{~g} / \mathrm{L}$ yeast extract, $0.4 \%(\mathrm{v} / \mathrm{v})$ glycerol, and $10 \%(\mathrm{v} / \mathrm{v})$ phosphate buffer (23.12 g/L KH $2 \mathrm{PO}_{4}$ and $\left.125.4 \mathrm{~g} / \mathrm{L} \mathrm{K}_{2} \mathrm{HPO}_{4}\right)$ ).

Construction of $P$. putida Tn7GFPstop Strain. To assess the efficiency levels of allelic replacement, easily selectable mutations had to be selected for our protocol. These mutations should have a visual phenotypic readout for easy screening, e.g., antibiotic resistance, change of color, or fluorescence emission. With this objective in mind, we generated $P$. putida Tn7GFPstop. This strain was created by introducing a $g f p$ gene cassette with a $g f p$ ORF disrupted by a TAG stop codon replacing Tyr66 (Supplementary Table S5), in the attTn7 landing site of the P. putida KT2440 genome. By mutating back the introduced TAG stop codon into the original sequence, the $g f p$ coding sequence would be restored and thus the strain would become fluorescent. The cassette was integrated following a previously described protocol for ISceI-mediated homologous recombination. ${ }^{75}$ In brief, the cassette was first amplified with primers M46 and M47 (Supplementary Table S3) from pSB1C3 disrupted gfp and cloned into the pGNW suicide vector (amplified with M40 and M41) between 500-bp upstream and downstream regions (amplified with M42-M43 and M44-M45, respectively) of the attTn7 landing site. $P$. putida KT2440 cells were transformed with pGNW via electroporation and resultant fluorescent colonies (cointegrates) were grown for pSEVA628 I-SceI vector transformation. Expression of I-SceI meganuclease was induced with 3-methylbenzoate mediating the excision of pGNW from the genome, leading to nonfluorescent colonies. Final clones were tested for revertant (i.e., wild type) or mutant (i.e., knock in) genotype.

Plasmids. Plasmids used in the present study are fully described in Supplementary Table S2. All PCR reactions for cloning purposes were performed with the NEB Q5 HighFidelity DNA polymerase, according to manufacturer's instructions (M0491). PCR fragments were subjected to $1 \%$ $\mathrm{w} / \mathrm{v}$ agarose gel electrophoresis, and isolated using Nucleospin Gel and PCR Clean-up (BIOKÉ) kit. Plasmids were built using the SevaBrick Assembly method, ${ }^{76}$ unless otherwise stated, and introduced by heat-shock in chemically competent E. coli DH5 $\alpha$ cells. Plasmids were isolated using the GeneJET Plasmid Miniprep Kit (Thermo Scientific) and colony PCR was performed to verify the right assembly of the different fragments. Plasmid sequence was confirmed by Sanger sequencing from Macrogen (MACROGEN Inc. DNA Sequencing Service; Amsterdam, The Netherlands).

Recombineering experiments were performed using pSEVA2514-rec2-mut $L_{\mathrm{E} 36 \mathrm{~K}}{ }^{\mathrm{PP}}$, pSEVA2514-rec2 RBSopt -mut$L_{\mathrm{E} 36 \mathrm{~K}}{ }^{\mathrm{PP}}$ RBSopt, pSEVA2514-paprecT-mut $L_{\mathrm{E} 36 \mathrm{~K}}{ }_{\mathrm{PP}}$, and pSEVA2514-paprec $T_{\mathrm{RBSopt}}-$-mut $_{\mathrm{E} 36 \mathrm{~K}}{ }_{\mathrm{PP}}{ }_{\mathrm{RBSopt}}$. The pSEVA2514rec2-mut $L_{\mathrm{E} 36 \mathrm{~K}}{ }_{\mathrm{PP}}$ plasmid was a kind gift from the Molecular Environmental Microbiology Laboratory (CNB-CSIC) of Madrid (GenBank \#MN180222) and was used as reference vector. The pSEVA2514-paprecT-mut $L_{\mathrm{E} 36 \mathrm{~K}}{ }^{\mathrm{PP}}$ plasmid was built via Gibson Assembly using NEBuilder HiFi DNA Assembly Master Mix by substituting rec2 with paprecT. The backbone was amplified with M70-M71 primers from the reference vector, and paprecT was amplified with M72-M73 primers from PORTMAGEE502B, which was purchased from Addgene (\#128971). Strengthening of RBSs for rec2, paprecT, and mut $L_{\mathrm{E} 36 \mathrm{~K}}{ }^{\mathrm{PP}}$ genes was done by predicting the strongest RBS upstream of the ORFs with the automated design tool De Novo $\mathrm{DNA}^{77}$ and can be found in Supplementary Table S5. Putatively optimal RBSs were incorporated in primers M76, 
M78, and M80, which were used together with M77, M79, and $\mathrm{M} 81$, to amplify rec2, paprec $T$, and $m u t L_{\mathrm{E} 36 \mathrm{~K}}{ }^{P P}$, respectively. $\mathrm{RBS}_{\text {opt }}$ amplicons were cloned in a three-part ligation (recombinase $+m u t L_{\mathrm{E} 36 \mathrm{~K}}{ }^{P P}$ ) into the linear backbone amplified with M74-M75 primers from the reference vector, for the construction of pSEVA2514-rec2 $2_{\mathrm{RBS}}-m u t L_{\mathrm{E} 36 \mathrm{~K}}{ }_{\mathrm{PP}}{ }_{\mathrm{RBSopt}}$ and pSEVA2514-paprec $T_{\mathrm{RBSopt}}-{ }^{-m u t} L_{\mathrm{E} 36 \mathrm{~K}}{ }_{\mathrm{PB}} \mathrm{RB}$ opt .

Fluorescence loss assays were performed using pSEVAb62ScCas9, pSEVAb23-RhaBAD-crRNA_sp and pSEVAb44sfGFP. The gene encoding the ScCas9 was codon optimized for P. putida using the Jcat codon optimization tool (www.jcat. de) (Supplementary Table S5). A pCCI-4k plasmid with the optimized ScCas9 gene was synthesized and delivered by GenScript. The pSEVAb62-ScCas9 plasmid was built by PCR amplifying the ScCas9 gene, tracrRNA and their respective promoters (cargo of the pCCI-4K plasmid) with Ep-Pp primers and cloning the fragment into a linearized pSEVAb62 backbone with Ev-Pv primers. The pSEVAb23-RhaBADcrRNA eforRed was built by using the pSB1C3-RhaBAD and pSEVAb23-crRNA_eforRed in-house plasmids. The pSB1C3-RhaBAD plasmid was used to amplify the rhamnose inducible promoter together with the activators rhaS and rhaR (rhaSR-PrhaBAD) with Ep-Sp primers. The pSEVAb23crRNA_eforRed plasmid was used to amplify the leader sequence and crRNA array with Xp-Pp primers. The crRNA is composed of two directed repeats interspaced by the transcriptional unit $\mathrm{P}_{\mathrm{J} 23100}-\mathrm{RBS}_{\mathrm{BBa} \_ \text {B0034-eforRed }} \mathrm{BBa}_{\mathrm{B} 592012}$, which, in turn, is flanked by two BsaI sites. ${ }^{78}$ The two PCR amplified fragments were cloned into a linearized pSEVAb23 backbone with Ev-Pv primers using the previously mentioned SevaBrick Assembly method with some modifications. The customary enzyme deactivation step at $80{ }^{\circ} \mathrm{C}$ was replaced by a process halting at $16^{\circ} \mathrm{C}$. The desired spacers were introduced in pSEVAb23-RhaBAD-crRNA_eforRed using the previously described protocol called One-step Golden Gate-based cloning for the assembly of single and multiple spacers into the crRNA cassette, $^{78}$ by replacing the eforRed chromoprotein (pSEVAb23-RhaBAD-crRNA_sp). The pSEVAb44-sfGFP plasmid was built by cloning the transcriptional unit, $\mathrm{P}_{\mathrm{J} 23106}$-RBS-sfGFP (amplified from pSB1C3-sfGFP in-house plasmid with Ep-Pp primers) into pSEVAb44 backbone (amplified with Ev-Pv primers).

Cleavage assays were performed using (i) two plasmid system: pSEVAb62-ScCas9/pSEVAb62-ScCas9 $\Delta$ loop and pSEVAb23-crRNA_sp and (ii) one plasmid system: pSEVAb62-ScCas9-crRNA_sp. The pSEVAb62-ScCas9 1 loop plasmid was built by removing the loop D367-376 from the ScCas9 using the pSEVAb62-ScCas9 plasmid as template. Two PCR fragments were created using 597-M160 and 594-M161 primers and ligated via SevaBrick Assembly.

The pSEVAb23-crRNA_amilCP plasmid was built using pSEVA231-CRISPR as template. ${ }^{28}$ The leader sequence and crRNA array were amplified with crRNA-F-crRNA-R primers. The translational unit, comprised by the $\mathrm{BBa} J 23100$ Anderson promoter $\left(\mathrm{P}_{\mathrm{J} 23100}\right)$, the BBa_B0034 RBS $\left(\mathrm{RBS}_{\mathrm{BBa}} \mathrm{B003}\right)$ and the amilCP, blue chromoprotein (BBa_K592009), was amplified with 804-940-primers from pSB1C3-amilCP in-house plasmid. The two PCR amplified fragments were cloned into a linearized pSEVAb23 backbone with 475-476 primers using the previously mentioned SevaBrick Assembly method with some modifications. The customary enzyme deactivation step at $80{ }^{\circ} \mathrm{C}$ was replaced by a process halting at $16{ }^{\circ} \mathrm{C}$. As result, the crRNA array is comprised by two directed repeats interspaced by the transcriptional unit $\mathrm{P}_{\mathrm{J} 23100}-\mathrm{RBS}_{\mathrm{BBa} \_ \text {B0034-amilCP }} \mathrm{BBa}_{78592009}$, which, in turn, is flanked by two BsaI sites. ${ }^{78}$ The pSEVAb62-ScCas9-crRNA_eforRed plasmid was built using the NEBuilder HiFi DNA Assembly Master Mix. A constitutive version of the $\mathrm{pGCRi}-\mathrm{R}^{78}$ was used to amplify the crRNA array $\left(\mathrm{P}_{\mathrm{J} 23100}-\mathrm{RBS}_{\mathrm{BBa} \text { B0034-eforRed }} \mathrm{BBa}\right.$ K592012 $)$ with M94-M95 primers. The PCR amplified crRNA array was cloned into pSEVAb62-ScCas9, linearized with M92-M93 primers. The desired spacers are introduced in pSEVAb23-crRNA_amilCP and pSEVAb62-ScCas9-crRNA_eforRed as previously described for the pSEVAb23-RhaBAD-crRNA_eforRed plasmid. All the spacers used in the present study can be found in Supplementary Table S4.

Oligonucleotides. Single-stranded (ss) DNA oligonucleotides employed in this study (Supplementary Table S3) were ordered from Integrated DNA technologies (IDT) as salt-free without further purification, resuspended in milli-Q at $100 \mu \mathrm{M}$ and long-term stored at $-20{ }^{\circ} \mathrm{C}$.

Recombineering oligos (Supplementary Table S3) were designed to be complementary to the lagging strand of replicating DNA and according to the optimized design criteria shown in the Supporting Information and Supplementary Figure S1. In sum, they were $60 \mathrm{nt}$ long and carried mutation changes at the middle positions of their DNA sequence; predicted folding energies were higher than $\geq 16 \mathrm{kcal} / \mathrm{mol}$, ${ }^{22}$ and no phosphorothioate bonds were included in the sequences.

Design of Optimized Recombineering Oligonucleotides. In order to optimize the design of recombineering oligonucleotides, different parameters were considered.

(i) Strandedness: Recombineering oligos were designed to anneal to the lagging strand of the replication fork since hybridization of ssDNA is supposed to occur there according to the principles of recombineering. ${ }^{21}$

(ii) Structure: ssDNA with higher predicted $\Delta G$ score is suggested to recombine at higher frequencies. ${ }^{39}$ While the optimal folding energy for $E$. coli has been reported to be $\sim 12.5 \mathrm{kcal} / \mathrm{mol}^{40}{ }^{40}$ the higher GC content and lower optimal growth temperature $\left(30{ }^{\circ} \mathrm{C}\right)$ suggest that the optimal range could be different for $P$. putida. ${ }^{22}$ According to the DNA folding predictor tool mfold-UNAFold, ${ }^{79}$ all the recombineering oligos used in this study had a folding energy $\geq 16 \mathrm{kcal} /$ mol.

(iii) Length: Oligonucleotides of 90, 60, and 40 nt were tested by using recombineering with the K43T mutation in the $r p s L$ gene that confers resistance to streptomycin. After the optimization study, the oligos were designed of $60 \mathrm{bp}$ in length, with the desired mutations included in the middle of the sequence.

(iv) Backbone modifications: For the oligonucleotide optimization study, oligos both with and without phosphorothioate bonds were tested with the K43T mutation in the rpsL gene. Accordingly, subsequent oligonucleotides did not include phosphorothioate bonds.

Recombineering Cycling Protocol. Recombineering experiments were performed according to the previously described standard protocol ${ }^{80}$ based on the coexpression of a recombinase and a mismatch repair machinery disruptor, both under the control of the thermoinducible $\mathrm{cI} 857 / \mathrm{P}_{\mathrm{L}}$ expression system. An overnight culture of $P$. putida KT2440 harboring pSEVA2514-recombinase ${ }^{*}-m u t L_{\mathrm{E} 36 \mathrm{~K}}{ }_{\mathrm{PP}}$ (* indicates any of the recombinases used in this work: Rec2, PapRecT and their 
respective RBS variations) was grown in $20 \mathrm{~mL}$ of $\mathrm{LB}$ supplemented with kanamycin. The next day, bacterial cultures were diluted to an $\mathrm{OD}_{600}$ of 0.1 in LB-kan and incubated at 30 ${ }^{\circ} \mathrm{C}, 200 \mathrm{rpm}$ until an $\mathrm{OD}_{600}$ of $0.5-0.7$ (mid log phase). Once the appropriate $\mathrm{OD}_{600}$ was reached, recombinase and mut $L_{\mathrm{E} 36 \mathrm{~K}}{ }^{\mathrm{PP}}$ transcription was thermoinduced by $10 \mathrm{~min}$ incubation at $42{ }^{\circ} \mathrm{C}$ in a shaking water bath. P. putida cultures were chilled on ice for $5 \mathrm{~min}$ and harvested by centrifugation at room temperature and $4700 \mathrm{~g}$ for $10 \mathrm{~min}$. Subsequently, cells were made electrocompetent by consecutive washing steps of 10,2 , and $1 \mathrm{~mL}$ of sucrose $300 \mathrm{mM}$. The washed cultures were finally resuspended in $200 \mu \mathrm{L}$ of sucrose $300 \mathrm{mM} .100 \mu \mathrm{L}$ of electrocompetent cells were transformed with $1 \mu \mathrm{L}$ of recombineering oligo $(100 \mu \mathrm{M})$. Samples in which CRISPRScCas9 counterselection was applied were transformed additionally with $100 \mathrm{ng}$ of pSEVAb62-ScCas9-crRNA_sp carrying the appropriate spacer, in addition to the corresponding recombineering oligonucleotide. Electroporation was performed in $2 \mathrm{~mm}$ gap Bio-Rad electroporation cuvettes. A single exponential decay pulse was applied using a Gene Pulser $\mathrm{X}$-Cell (Bio-Rad) set at $2.5 \mathrm{kV}, 200 \Omega$ and $25 \mu \mathrm{F}$. Cells were first resuspended in $5 \mathrm{~mL}$ of terrific broth (TB) with kanamycin and recovered for $1 \mathrm{~h}$. Afterward, $15 \mathrm{~mL}$ of LBkan was added to the transformed cells. Cultures were grown to an $\mathrm{OD}_{600}$ of $\sim 0.4$, and stored at $4{ }^{\circ} \mathrm{C}$ until the next day. In consecutive cycles, cultures stored at $4{ }^{\circ} \mathrm{C}$ were reactivated by $\sim 30 \mathrm{~min}$ incubation at $30{ }^{\circ} \mathrm{C}$ and $200 \mathrm{rpm}$ until an $\mathrm{OD}_{600}$ of $0.5-0.7$ before continuing with subsequent recombinase and $m u t L_{\mathrm{E} 36 \mathrm{~K}}{ }^{\mathrm{PP}}$ induction.

Before storing the cultures at $4{ }^{\circ} \mathrm{C}, 1 \mathrm{~mL}$ of the bacterial cells with $\mathrm{OD}_{600}$ of $\sim 0.4$ from cycles $1,4,7$, and 10 was inoculated into $2 \mathrm{~mL} \mathrm{LB}-\mathrm{kan}$ and grown at $30^{\circ} \mathrm{C}$ and $200 \mathrm{rpm}$, overnight. Appropriate dilutions from the overnight cultures were plated for screening and subsequent efficiency calculation. Cultures without thermoinduction were included as negative controls. Editing efficiencies of non-thermoinduced controls were subtracted from those of the thermoinduced samples to calculate final recombineering efficiencies as it is assumed that such background levels are not directly derived from the action of the tested recombinases. ${ }^{25}$

Screening of Cells Edited with Recombineering. On the basis of the phenotypic outcome of the introduced mutations, three different readouts and screening methods were analyzed in this study.

First, P. putida KT2440 clones edited in the rpsL gene (PP_0449) were engineered with oligo RO rpsL 60 for the change of the AAA codon (Lys43) by ACA (Thr43) and were screened by the resistant to streptomycin conferred by this change. Recombineering efficiency was calculated as the ratio between streptomycin resistant and total CFUs. Screening was performed using both LB and LB-sm plates. $^{23}$

Second, P. putida Tn7GFPstop clones edited in a heterologous and disrupted $g f p$ gene were engineered with oligo RO gfp stop in order to revert the functional expression of the green fluorescence reporter (Stop66Tyr). Recombineering efficiency was calculated as the ratio between fluorescent (mutated) and total CFUs. Screening was performed in LBkan agar plates after $\sim 48 \mathrm{~h}$, allowing GFP maturation.

Lastly, efficiencies of recombinant cells without screenable or selectable phenotypes (TAG $\rightarrow$ TAA mutants) were determined by multiplex allele-specific colony PCR (MASCPCR $)^{16,40}$ or high-resolution melt analysis (HRM). ${ }^{48,49}$ For MASC-PCR, three primers were designed for each targeted locus: (i) FW primer specific to the wild type genotype, (ii) FW primers specific for the mutant genotype, and (iii) RV primer common to both. The two FW primers only differed at their 3'-terminal bases allowing discrimination of single nucleotide changes. Two MASC-PCR reactions were required to screen each colony: one to test the wild type genotype and one to test the mutant genotype. Colony genotype was therefore revealed by the binary result yielded by the two reactions. When possible, several loci were interrogated in a single reaction by designing different primer sets with the same melting temperature but different amplicon length. In this case, primer pools were used for the PCR reactions. For HRM, specific primers for $100 \mathrm{bp}$ amplicons were used for colony PCR supplemented with LCGreen Plus+ Melting Dye. Since exact melting temperatures of DNA molecules are determined by their nucleic acid sequence, differences between amplicon samples even with only one single nucleotide variation result in melting profiles that are unique to these particular genotypes, allowing for differentiation between amplicons containing the TAG WT and the TAA mutant genotypes. After amplification, samples were transferred to a LightScanner Instrument (BIOKÉ) for melting and acquisition of melt curves, and subsequent data analysis was performed by the LightScanner software. Both types, MASC and HRM colony PCRs, were performed with Phire Hot start II DNA Polymerase (Thermo Fisher Scientific) according to manufacturer's guidelines. Recombineering efficiency was ultimately calculated as the ratio between mutants and total CFUs.

Fluorescence Loss Assays. P. putida KT2440 $\left(\operatorname{rec}^{+}\right)$, harboring pSEVAb62-ScCas9, pSEVAb23-RhaBAD-crRNA_sp with the desired spacer and pSEVAb44-sfGFP, were grown at $30{ }^{\circ} \mathrm{C}$ and $200 \mathrm{rpm}$, overnight in $10 \mathrm{~mL} \mathrm{LB}$ media supplemented with kanamycin, gentamycin, and streptomycin. Overnight cells were harvested at $4700 \mathrm{~g}$ for $10 \mathrm{~min}$ and washed with minimal M9 medium in order to eliminate LB traces. Cells were resuspended to an $\mathrm{OD}_{600}$ of 0.3 and grown aerobically at $30{ }^{\circ} \mathrm{C}$ in fresh minimal M9 medium supplemented with $70 \mathrm{mM}$ of glucose and the appropriated antibiotics (kanamycin and gentamicin at 50 and $10 \mu \mathrm{g} / \mathrm{mL}$, respectively) on 96-well black wall and transparent roundbottom plate in a total volume of $200 \mu \mathrm{L}$ per well. Additionally, $5 \mathrm{mM}$ of L-rhamnose was added to the media under induced conditions. Optical density $\left(\mathrm{OD}_{600}\right)$ and green fluorescence (excitation $467 \mathrm{~nm}$, emission $508 \mathrm{~nm}$ ) readings were monitored in a BioTek Synergy Mx Multi-Mode Microplate reader over $24 \mathrm{~h}$. Fluorescence values were normalized to $\mathrm{OD}_{600}$ values. Biological and technical triplicates were included.

Cleavage Assays. Strains P. putida KT2440 $\left(\operatorname{rec} A^{+}\right)$, P. putida EM383 $\left(\operatorname{rec} A^{-}\right)$, E. coli BL21 $\left(\operatorname{rec} A^{+}\right)$and E. coli DH5 $\alpha\left(r e c A^{-}\right)$, harboring pSEVAb62-ScCas9 or pSEVAb62ScCas9 9 loop were used in the cleavage assays based on twoplasmid system. Electrocompetent $P$. putida and chemicalcompetent E. coli cells were transformed with $100 \mathrm{ng}$ of pSEVAb23-crRNA_sp (with different targeting spacers) and plated in LB-Kan-Gen solid agar media. The pSEVAb23crRNA_nt plasmid harbors a non-targeting spacer that does not target any region in the genome and was used as control. Strains P. putida KT2440 (recA $\left.{ }^{+}\right)$and P. putida EM383 (rec $\left.A^{-}\right)$ were used in the cleavage assays based on one-plasmid system. pSEVAb62-ScCas9-crRNA sp with non-targeting spacer (control) and targeting spacers were transformed in electrocompetent $P$. putida strains. Targeting efficiency for both 
strategies was calculated by as the percentage of surviving CFUs present in plates transformed with targeting spacers divided by the number of CFUs present in plates transformed with the non-targeting spacer. All transformations were repeated at least two times.

Fitness and Toxicity Assays. To measure the fitness of the strains and the toxicity of ScCas9 for P. putida, growth assays were conducted in LB and M9-glucose media in an Elx808 Absorbance Microplate Reader (BioTek Instruments, Inc., VT, U.S.). Optical density at $600 \mathrm{~nm}$ was monitored for $24 \mathrm{~h}$ after seeding with $200 \mu \mathrm{L}$ cultures at $\mathrm{OD}_{600}=0.1$.

Whole-Genome Sequencing. In order to confirm the mutated loci of $P$. putida KT2440Rc12 and to measure offtarget mutagenesis, gDNA of the strain was isolated by using the GenElute Bacterial Genomic DNA kit (Sigma-Aldrich). Extracted gDNA was sent for sequencing to Novogene Co. Ltd. (Beijing, China) for Illumina sequencing. Raw lllumina reads were trimmed for low quality and adapters with fastp (v0.20.0). Mutations were found using breseq (v0.35.5) using the reference genome and annotation of Pseudomonas putida KT2440 (GCF_000007565.2). To calculate the number of offtarget mutations, the total number of nonintended mutations was divided by the number of recombineering cycles performed in the KT2440Rc12 strain, after removing all those mutations also present in the sequence of an in-house reference strain that was subjected to the same whole-genome sequencing and analysis.

\section{ASSOCIATED CONTENT}

\section{SI Supporting Information}

The Supporting Information is available free of charge at https://pubs.acs.org/doi/10.1021/acssynbio.1c00297.

(a) Supplementary Figure S1: Fluorescence loss assays, (b) Supplementary Table S1: Strains, (c) Supplementary Table S2: Plasmids, (d) Supplementary Table S3: Oligonucleotides (spacers, PCR amplification primers, MASC-PCR primers and recombineering oligonucleotides), (e) Supplementary Table S4: Guide RNAs, (f) Supplementary Table S5: Additional DNA sequences, (g) Supplementary Table S6: List of P. putida KT2440 genes containing TAG stop codon and TAG genomic coordinates and characteristics, (h) Supplementary Table S7: FBA predicted metabolic essential genes of $P$. putida KT2440 in glucose minimal media with their respective stop codons, (i) Supplementary Table S8: Efficiencies of the different experiments of ReScribe including single, duplex, and triplex, and (j) Supplementary Table S9: Data of the whole-genome sequencing of $P$. putida KT2440Rc12 strain (PDF)

Spreadsheet versions of Tables S1-S9 (XLSX)

\section{AUTHOR INFORMATION}

\section{Corresponding Author}

Vitor A. P. Martins dos Santos - Laboratory of Systems and Synthetic Biology, Wageningen University \& Research, Wageningen 6708 WE, The Netherlands; LifeGlimmer $\mathrm{GmbH}$, Berlin 12163, Germany; Bioprocess Engineering Group, Wageningen University \& Research, Wageningen 6700 AA, The Netherlands; (1) orcid.org/0000-0002-23529017; Phone: +31317482865;

Email: vitor.martinsdossantos@wur.nl
Authors

Enrique Asin-Garcia - Laboratory of Systems and Synthetic Biology, Wageningen University \& Research, Wageningen 6708 WE, The Netherlands

Maria Martin-Pascual - Laboratory of Systems and Synthetic Biology, Wageningen University \& Research, Wageningen 6708 WE, The Netherlands

Luis Garcia-Morales - Laboratory of Systems and Synthetic Biology, Wageningen University \& Research, Wageningen 6708 WE, The Netherlands

Richard van Kranenburg - Corbion, Gorinchem 4206 AC, The Netherlands; Laboratory of Microbiology, Wageningen University \& Research, Wageningen 6708 WE, The Netherlands

Complete contact information is available at:

https://pubs.acs.org/10.1021/acssynbio.1c00297

\section{Author Contributions}

\#EAG and MMP are joint first authors.

\section{Author Contributions}

Conceptualization: EAG, MMP, VMdS. Data curation: EAG, MMP. Formal analysis: EAG, MMP. Funding acquisition: MMP, RvK, VMdS. Investigation: EAG, MMP. Methodology: EAG, MMP. Project administration: VMdS. Resources: VMdS. Software: EAG, MMP. Supervision: LGM, RvK, VMdS. Validation: EAG, MMP. Visualization: EAG, MMP. Writing (original draft): EAG, MMP. Writing (review and editing): EAG, MMP, LGM, RvK, VMdS.

Notes

The authors declare no competing financial interest.

\section{ACKNOWLEDGMENTS}

We would like to thank Víctor de Lorenzo, Tomás Aparicio, and Esteban Martínez-García for providing the reference vector pSEVA2514-rec2-mut $L_{\mathrm{E} 36 \mathrm{~K}}{ }^{\mathrm{PP}}$ and, most importantly, for their valuable advice and comments throughout the different stages of this study. We wish to convey our gratitude as well to Farren Isaacs for the feedback and suggestions within the project; to Christos Batianis for his help with the P. putida genome scale, constraint-based metabolic model iJP962, and the useful conversations about this work; and to Lorenzo Olivi for his guidance with Python and Artemis. We also thank Daniel Moñino-López for discussing some ingenious ideas that have materialized on some of the key points of this paper. This work is part of the research programs SafeChassis: Implementing and Assessing Safeguards for Lifestyle Engineering of a Versatile Industrial Chassis and Putida for Plastics, both supported by the Dutch Research Council (NWO) [15814, GSGT.2019.028]. Funding for open access charge: Dutch Research Council.

\section{REFERENCES}

(1) Poblete-Castro, I.; Becker, J.; Dohnt, K.; dos Santos, V. M.; Wittmann, C. Industrial Biotechnology of Pseudomonas putida and Related Species. Appl. Microbiol. Biotechnol. 2012, 93 (6), 2279-2290.

(2) Nikel, P. I.; de Lorenzo, V. Pseudomonas putida as a Functional Chassis for Industrial Biocatalysis: From Native Biochemistry to Trans-Metabolism. Metab. Eng. 2018, 50, 142-155.

(3) Calero, P.; Nikel, P. I. Chasing Bacterial Chassis for Metabolic Engineering: A Perspective Review from Classical to Non-Traditional Microorganisms. Microb. Biotechnol. 2019, 12 (1), 98-124.

(4) Martin-Pascual, M.; Batianis, C.; Bruinsma, L.; Asin-Garcia, E.; Garcia-Morales, L.; Weusthuis, R. A.; van Kranenburg, R.; Martins 
dos Santos, V. A. P. A Navigation Guide of Synthetic Biology Tools for Pseudomonas putida. Biotechnol. Adv. 2021, 49, 107732.

(5) Lau, Y. H.; Stirling, F.; Kuo, J.; Karrenbelt, M. A. P.; Chan, Y. A.; Riesselman, A.; Horton, C. A.; Schäfer, E.; Lips, D.; Weinstock, M. T.; Gibson, D. G.; Way, J. C.; Silver, P. A. Large-Scale Recoding of a Bacterial Genome by Iterative Recombineering of Synthetic DNA. Nucleic Acids Res. 2017, 45 (11), 6971-6980.

(6) Chin, J. W. Reprogramming the Genetic Code. EMBO J. 2011, 30 (12), 2312-2324.

(7) Nikel, P. I. Synthesis of Recoded Bacterial Genomes toward Bespoke Biocatalysis. Trends Biotechnol. 2019, 37 (10), 1036-1038.

(8) Lajoie, M. J.; Rovner, A. J.; Goodman, D. B.; Aerni, H.-R.; Haimovich, A. D.; Kuznetsov, G.; Mercer, J. A.; Wang, H. H.; Carr, P. A.; Mosberg, J. A.; Rohland, N.; Schultz, P. G.; Jacobson, J. M.; Rinehart, J.; Church, G. M.; Isaacs, F. J. Genomically Recoded Organisms Expand Biological Functions. Science 2013, 342 (6156), 357-360.

(9) Ma, N. J.; Isaacs, F. J. Genomic Recoding Broadly Obstructs the Propagation of Horizontally Transferred Genetic Elements. Cell Systems 2016, 3 (2), 199-207.

(10) Rovner, A. J.; Haimovich, A. D.; Katz, S. R.; Li, Z.; Grome, M. W.; Gassaway, B. M.; Amiram, M.; Patel, J. R.; Gallagher, R. R.; Rinehart, J.; Isaacs, F. J. Recoded Organisms Engineered to Depend on Synthetic Amino Acids. Nature 2015, 518 (7537), 89-93.

(11) Martínez, M. A.; Jordan-Paiz, A.; Franco, S.; Nevot, M. Synonymous Virus Genome Recoding as a Tool to Impact Viral Fitness. Trends Microbiol. 2016, 24 (2), 134-147.

(12) Richardson, S. M.; Mitchell, L. A.; Stracquadanio, G.; Yang, K.; Dymond, J. S.; DiCarlo, J. E.; Lee, D.; Huang, C. L. V.; Chandrasegaran, S.; Cai, Y.; Boeke, J. D.; Bader, J. S. Design of a Synthetic Yeast Genome. Science 2017, 355 (6329), 1040-1044.

(13) Wang, K.; Fredens, J.; Brunner, S. F.; Kim, S. H.; Chia, T.; Chin, J. W. Defining Synonymous Codon Compression Schemes by Genome Recoding. Nature 2016, 539 (7627), 59-64.

(14) Fredens, J.; Wang, K.; de la Torre, D.; Funke, L. F. H.; Robertson, W. E.; Christova, Y.; Chia, T.; Schmied, W. H.; Dunkelmann, D. L.; Beránek, V.; Uttamapinant, C.; Llamazares, A. G.; Elliott, T. S.; Chin, J. W. Total Synthesis of Escherichia coli with a Recoded Genome. Nature 2019, 569 (7757), 514-518.

(15) Kuo, J.; Stirling, F.; Lau, Y. H.; Shulgina, Y.; Way, J. C.; Silver, P. A. Synthetic Genome Recoding: New Genetic Codes for New Features. Curr. Genet. 2018, 64 (2), 327-333.

(16) Isaacs, F. J.; Carr, P. A.; Wang, H. H.; Lajoie, M. J.; Sterling, B.; Kraal, L.; Tolonen, A. C.; Gianoulis, T. A.; Goodman, D. B.; Reppas, N. B.; Emig, C. J.; Bang, D.; Hwang, S. J.; Jewett, M. C.; Jacobson, J. M.; Church, G. M. Precise Manipulation of Chromosomes in Vivo Enables Genome-Wide Codon Replacement. Science 2011, 333 (6040), 348-353.

(17) Mandell, D. J.; Lajoie, M. J.; Mee, M. T.; Takeuchi, R.; Kuznetsov, G.; Norville, J. E.; Gregg, C. J.; Stoddard, B. L.; Church, G. M. Biocontainment of Genetically Modified Organisms by Synthetic Protein Design. Nature 2015, 518 (7537), 55-60.

(18) Ostrov, N.; Landon, M.; Guell, M.; Kuznetsov, G.; Teramoto, J.; Cervantes, N.; Zhou, M.; Singh, K.; Napolitano, M. G.; Moosburner, M.; Shrock, E.; Pruitt, B. W.; Conway, N.; Goodman, D. B.; Gardner, C. L.; Tyree, G.; Gonzales, A.; Wanner, B. L.; Norville, J. E.; Lajoie, M. J.; Church, G. M. Design, Synthesis, and Testing toward a 57-Codon Genome. Science 2016, 353 (6301), 819822.

(19) Hutchison, C. A.; Chuang, R.-Y.; Noskov, V. N.; Assad-Garcia, N.; Deerinck, T. J.; Ellisman, M. H.; Gill, J.; Kannan, K.; Karas, B. J.; Ma, L.; Pelletier, J. F.; Qi, Z.-Q.; Richter, R. A.; Strychalski, E. A.; Sun, L.; Suzuki, Y.; Tsvetanova, B.; Wise, K. S.; Smith, H. O.; Glass, J. I.; Merryman, C.; Gibson, D. G.; Venter, J. C. Design and Synthesis of a Minimal Bacterial Genome. Science 2016, 351 (6280), aad6253.

(20) Murphy, K. C. $\lambda$ Recombination and Recombineering. EcoSal Plus 2016, DOI: 10.1128/ecosalplus.ESP-0011-2015.

(21) Ellis, H. M.; Yu, D.; DiTizio, T.; Court, D. L. High Efficiency Mutagenesis, Repair, and Engineering of Chromosomal DNA Using
Single-Stranded Oligonucleotides. Proc. Natl. Acad. Sci. U. S. A. 2001, 98 (12), 6742-6746.

(22) Aparicio, T.; Jensen, S. I.; Nielsen, A. T.; de Lorenzo, V.; Martínez-García, E. The Ssr Protein (T1E_1405) from Pseudomonas Putida DOT-T1E Enables Oligonucleotide-Based Recombineering in Platform Strain P. Putida EM42. Biotechnol. J. 2016, 11 (10), 13091319.

(23) Ricaurte, D. E.; Martínez-García, E.; Nyerges, Á.; Pál, C.; de Lorenzo, V.; Aparicio, T. A Standardized Workflow for Surveying Recombinases Expands Bacterial Genome-Editing Capabilities. Microb. Biotechnol. 2018, 11 (1), 176-188.

(24) Nyerges, Á.; Csörgő, B.; Nagy, I.; Bálint, B.; Bihari, P.; Lázár, V.; Apjok, G.; Umenhoffer, K.; Bogos, B.; Pósfai, G.; Pál, C. A Highly Precise and Portable Genome Engineering Method Allows Comparison of Mutational Effects across Bacterial Species. Proc. Natl. Acad. Sci. U. S. A. 2016, 113 (9), 2502-2507.

(25) Aparicio, T.; Nyerges, A.; Martínez-García, E.; de Lorenzo, V. High-Efficiency Multi-Site Genomic Editing of Pseudomonas putida through Thermoinducible SsDNA Recombineering. iScience 2020, 23 (3), 100946.

(26) Wannier, T. M.; Nyerges, A.; Kuchwara, H. M.; Czikkely, M.; Balogh, D.; Filsinger, G. T.; Borders, N. C.; Gregg, C. J.; Lajoie, M. J.; Rios, X.; Pál, C.; Church, G. M. Improved Bacterial Recombineering by Parallelized Protein Discovery. Proc. Natl. Acad. Sci. U. S. A. 2020, 117 (24), 13689-13698.

(27) Selle, K.; Barrangou, R. CRISPR-Based Technologies and the Future of Food Science. J. Food Sci. 2015, 80 (11), R2367-2372.

(28) Aparicio, T.; de Lorenzo, V.; Martínez-García, E. CRISPR/ Cas9-Based Counterselection Boosts Recombineering Efficiency in Pseudomonas putida. Biotechnol. J. 2018, 13 (5), 1700161.

(29) Jiang, W.; Bikard, D.; Cox, D.; Zhang, F.; Marraffini, L. A. RNA-Guided Editing of Bacterial Genomes Using CRISPR-Cas Systems. Nat. Biotechnol. 2013, 31 (3), 233-239.

(30) Chatterjee, P.; Jakimo, N.; Jacobson, J. M. Minimal PAM Specificity of a Highly Similar SpCas9 Ortholog. Science Advances 2018, 4 (10), eaau0766.

(31) Nelson, K. E.; Weinel, C.; Paulsen, I. T.; Dodson, R. J.; Hilbert, H.; Martins dos Santos, V. a. P.; Fouts, D. E.; Gill, S. R.; Pop, M.; Holmes, M.; Brinkac, L.; Beanan, M.; DeBoy, R. T.; Daugherty, S.; Kolonay, J.; Madupu, R.; Nelson, W.; White, O.; Peterson, J.; Khouri, H.; Hance, I.; Chris Lee, P.; Holtzapple, E.; Scanlan, D.; Tran, K.; Moazzez, A.; Utterback, T.; Rizzo, M.; Lee, K.; Kosack, D.; Moestl, D.; Wedler, H.; Lauber, J.; Stjepandic, D.; Hoheisel, J.; Straetz, M.; Heim, S.; Kiewitz, C.; Eisen, J. A.; Timmis, K. N.; Düsterhöft, A.; Tümmler, B.; Fraser, C. M. Complete Genome Sequence and Comparative Analysis of the Metabolically Versatile Pseudomonas putida KT2440. Environ. Microbiol. 2002, 4 (12), 799-808.

(32) Povolotskaya, I. S.; Kondrashov, F. A.; Ledda, A.; Vlasov, P. K. Stop Codons in Bacteria Are Not Selectively Equivalent. Biol. Direct 2012, 7 (1), 30.

(33) Korkmaz, G.; Holm, M.; Wiens, T.; Sanyal, S. Comprehensive Analysis of Stop Codon Usage in Bacteria and Its Correlation with Release Factor Abundance*. J. Biol. Chem. 2014, 289 (44), 3033430342.

(34) Poulsen, B. E.; Yang, R.; Clatworthy, A. E.; White, T.; Osmulski, S. J.; Li, L.; Penaranda, C.; Lander, E. S.; Shoresh, N.; Hung, D. T. Defining the Core Essential Genome of Pseudomonas aeruginosa. Proc. Natl. Acad. Sci. U. S. A. 2019, 116 (20), 1007210080 .

(35) Wetmore, K. M.; Price, M. N.; Waters, R. J.; Lamson, J. S.; He, J.; Hoover, C. A.; Blow, M. J.; Bristow, J.; Butland, G.; Arkin, A. P.; Deutschbauer, A. Rapid Quantification of Mutant Fitness in Diverse Bacteria by Sequencing Randomly Bar-Coded Transposons. mBio 2015, DOI: $10.1128 / \mathrm{mBio} .00306-15$.

(36) Belda, E.; van Heck, R. G. A.; Lopez-Sanchez, M. J.; Cruveiller, S.; Barbe, V.; Fraser, C.; Klenk, H.-P.; Petersen, J.; Morgat, A.; Nikel, P. I.; Vallenet, D.; Rouy, Z.; Sekowska, A.; dos Santos, V. A. P. M.; de Lorenzo, V.; Danchin, A.; Médigue, C. The Revisited Genome of 
Pseudomonas putida KT2440 Enlightens Its Value as a Robust Metabolic Chassis. Environ. Microbiol. 2016, 18 (10), 3403-3424.

(37) Puchałka, J.; Oberhardt, M. A.; Godinho, M.; Bielecka, A.; Regenhardt, D.; Timmis, K. N.; Papin, J. A.; Martins dos Santos, V. A. P. Genome-Scale Reconstruction and Analysis of the Pseudomonas putida KT2440 Metabolic Network Facilitates Applications in Biotechnology. PLoS Comput. Biol. 2008, 4 (10), e1000210.

(38) Molina-Henares, M. A.; de la Torre, J.; García-Salamanca, A.; Molina-Henares, A. J.; Herrera, M. C.; Ramos, J. L.; Duque, E. Identification of Conditionally Essential Genes for Growth of Pseudomonas Putida KT2440 on Minimal Medium through the Screening of a Genome-Wide Mutant Library. Environ. Microbiol. 2010, 12 (6), 1468-1485.

(39) Wang, H. H.; Isaacs, F. J.; Carr, P. A.; Sun, Z. Z.; Xu, G.; Forest, C. R.; Church, G. M. Programming Cells by Multiplex Genome Engineering and Accelerated Evolution. Nature 2009, 460 (7257), 894-898.

(40) Gallagher, R. R.; Li, Z.; Lewis, A. O.; Isaacs, F. J. Rapid Editing and Evolution of Bacterial Genomes Using Libraries of Synthetic DNA. Nat. Protoc. 2014, 9 (10), 2301-2316.

(41) Aparicio, T.; de Lorenzo, V.; Martínez-García, E. Improved Thermotolerance of Genome-Reduced Pseudomonas putida EM42 Enables Effective Functioning of the PL/CI857 System. Biotechnol. J. 2019, 14 (1), 1800483

(42) Chang, Y.; Wang, Q.; Su, T.; Qi, Q. The Efficiency for Recombineering Is Dependent on the Source of the Phage Recombinase Function Unit. bioRxiv, August 24, 2019. DOI: $10.1101 / 745448$.

(43) Cui, L.; Bikard, D. Consequences of Cas9 Cleavage in the Chromosome of Escherichia Coli. Nucleic Acids Res. 2016, 44 (9), $4243-4251$.

(44) Bikard, D.; Hatoum-Aslan, A.; Mucida, D.; Marraffini, L. A. CRISPR Interference Can Prevent Natural Transformation and Virulence Acquisition during in Vivo Bacterial Infection. Cell Host Microbe 2012, 12 (2), 177-186.

(45) Komor, A. C.; Badran, A. H.; Liu, D. R. CRISPR-Based Technologies for the Manipulation of Eukaryotic Genomes. Cell 2017, 168 (1), 20-36.

(46) Qi, L. S.; Larson, M. H.; Gilbert, L. A.; Doudna, J. A.; Weissman, J. S.; Arkin, A. P.; Lim, W. A. Repurposing CRISPR as an RNA-Guided Platform for Sequence-Specific Control of Gene Expression. Cell 2013, 152 (5), 1173-1183.

(47) Peters, J. M.; Colavin, A.; Shi, H.; Czarny, T. L.; Larson, M. H.; Wong, S.; Hawkins, J. S.; Lu, C. H. S.; Koo, B.-M.; Marta, E.; Shiver, A. L.; Whitehead, E. H.; Weissman, J. S.; Brown, E. D.; Qi, L. S.; Huang, K. C.; Gross, C. A. A Comprehensive, CRISPR-Based Functional Analysis of Essential Genes in Bacteria. Cell 2016, 165 (6), $1493-1506$.

(48) Wittwer, C. T. High-Resolution DNA Melting Analysis: Advancements and Limitations. Hum. Mutat. 2009, 30 (6), 857-859.

(49) Song, M.; Li, J.; Xiong, C.; Liu, H.; Liang, J. Applying HighResolution Melting (HRM) Technology to Identify Five Commonly Used Artemisia Species. Sci. Rep. 2016, 6 (1), 34133.

(50) Abbasi, M. N.; Fu, J.; Bian, X.; Wang, H.; Zhang, Y.; Li, A. Recombineering for Genetic Engineering of Natural Product Biosynthetic Pathways. Trends Biotechnol. 2020, 38 (7), 715-728.

(51) Sun, Z.; Deng, A.; Hu, T.; Wu, J.; Sun, Q.; Bai, H.; Zhang, G.; Wen, T. A High-Efficiency Recombineering System with PCR-Based SsDNA in Bacillus Subtilis Mediated by the Native Phage Recombinase GP35. Appl. Microbiol. Biotechnol. 2015, 99 (12), $5151-5162$

(52) Binder, S.; Siedler, S.; Marienhagen, J.; Bott, M.; Eggeling, L. Recombineering in Corynebacterium glutamicum Combined with Optical Nanosensors: A General Strategy for Fast Producer Strain Generation. Nucleic Acids Res. 2013, 41 (12), 6360-6369.

(53) Guo, T.; Xin, Y.; Zhang, Y.; Gu, X.; Kong, J. A Rapid and Versatile Tool for Genomic Engineering in Lactococcus lactis. Microb. Cell Fact. 2019, 18 (1), 22.
(54) van Pijkeren, J.-P.; Britton, R. A. High Efficiency Recombineering in Lactic Acid Bacteria. Nucleic Acids Res. 2012, 40 (10), e76.

(55) Penewit, K.; Holmes, E. A.; McLean, K.; Ren, M.; Waalkes, A.; Salipante, S. J. Efficient and Scalable Precision Genome Editing in Staphylococcus aureus through Conditional Recombineering and CRISPR/Cas9-Mediated Counterselection. mBio 2018 , DOI: $10.1128 / \mathrm{mBio} .00067-18$.

(56) Zhao, D.; Feng, X.; Zhu, X.; Wu, T.; Zhang, X.; Bi, C. CRISPR/ Cas9-Assisted GRNA-Free One-Step Genome Editing with No Sequence Limitations and Improved Targeting Efficiency. Sci. Rep. 2017, 7 (1), 16624.

(57) Torkelson, J.; Harris, R. S.; Lombardo, M. J.; Nagendran, J.; Thulin, C.; Rosenberg, S. M. Genome-Wide Hypermutation in a Subpopulation of Stationary-Phase Cells Underlies RecombinationDependent Adaptive Mutation. EMBO J. 1997, 16 (11), 3303-3311.

(58) Sobetzko, P.; Travers, A.; Muskhelishvili, G. Gene Order and Chromosome Dynamics Coordinate Spatiotemporal Gene Expression during the Bacterial Growth Cycle. Proc. Natl. Acad. Sci. U. S. A. 2012, 109 (2), 355-356.

(59) Pines, G.; Freed, E. F.; Winkler, J. D.; Gill, R. T. Bacterial Recombineering: Genome Engineering via Phage-Based Homologous Recombination. ACS Synth. Biol. 2015, 4 (11), 1176-1185.

(60) Filsinger, G. T.; Wannier, T. M.; Pedersen, F. B.; Lutz, I. D.; Zhang, J.; Stork, D. A.; Debnath, A.; Gozzi, K.; Kuchwara, H.; Volf, V.; Wang, S.; Rios, X.; Gregg, C. J.; Lajoie, M. J.; Shipman, S. L.; Aach, J.; Laub, M. T.; Church, G. M. Characterizing the Portability of Phage-Encoded Homologous Recombination Proteins. Nat. Chem. Biol. 2021, 17, 394-402.

(61) Caldwell, B. J.; Zakharova, E.; Filsinger, G. T.; Wannier, T. M.; Hempfling, J. P.; Chun-Der, L.; Pei, D.; Church, G. M.; Bell, C. E. Crystal Structure of the $\operatorname{Red} \beta$ C-Terminal Domain in Complex with $\lambda$ Exonuclease Reveals an Unexpected Homology with $\lambda$ Orf and an Interaction with Escherichia Coli Single Stranded DNA Binding Protein. Nucleic Acids Res. 2019, 47 (4), 1950-1963.

(62) Yin, J.; Zheng, W.; Gao, Y.; Jiang, C.; Shi, H.; Diao, X.; Li, S.; Chen, H.; Wang, H.; Li, R.; Li, A.; Xia, L.; Yin, Y.; Stewart, A. F.; Zhang, Y.; Fu, J. Single-Stranded DNA-Binding Protein and Exogenous RecBCD Inhibitors Enhance Phage-Derived Homologous Recombination in Pseudomonas. iScience 2019, 14, 1-14.

(63) Creutzburg, S. C. A.; Wu, W. Y.; Mohanraju, P.; Swartjes, T.; Alkan, F.; Gorodkin, J.; Staals, R. H. J.; van der Oost, J. Good Guide, Bad Guide: Spacer Sequence-Dependent Cleavage Efficiency of Cas12a. Nucleic Acids Res. 2020, 48 (6), 3228-3243.

(64) Azpiroz, M. F.; Laviña, M. Analysis of RecA-Independent Recombination Events between Short Direct Repeats Related to a Genomic Island and to a Plasmid in Escherichia coli K12. PeerJ 2017, 5, e3293.

(65) Chatterjee, P.; Jakimo, N.; Lee, J.; Amrani, N.; Rodríguez, T.; Koseki, S. R. T.; Tysinger, E.; Qing, R.; Hao, S.; Sontheimer, E. J.; Jacobson, J. An Engineered ScCas9 with Broad PAM Range and High Specificity and Activity. Nat. Biotechnol. 2020, 38 (10), 1154-1158.

(66) Wannier, T. M.; Ciaccia, P. N.; Ellington, A. D.; Filsinger, G. T.; Isaacs, F. J.; Javanmardi, K.; Jones, M. A.; Kunjapur, A. M.; Nyerges, A.; Pal, C.; Schubert, M. G.; Church, G. M. Recombineering and MAGE. Nat. Rev. Methods Primers 2021, 1 (1), 1-24.

(67) Gibson, D. G.; Benders, G. A.; Andrews-Pfannkoch, C.; Denisova, E. A.; Baden-Tillson, H.; Zaveri, J.; Stockwell, T. B.; Brownley, A.; Thomas, D. W.; Algire, M. A.; Merryman, C.; Young, L.; Noskov, V. N.; Glass, J. I.; Venter, J. C.; Hutchison, C. A.; Smith, H. O. Complete Chemical Synthesis, Assembly, and Cloning of a Mycoplasma Genitalium Genome. Science 2008, 319 (5867), 12151220.

(68) Gibson, D. G.; Glass, J. I.; Lartigue, C.; Noskov, V. N.; Chuang, R.-Y.; Algire, M. A.; Benders, G. A.; Montague, M. G.; Ma, L.; Moodie, M. M.; Merryman, C.; Vashee, S.; Krishnakumar, R.; AssadGarcia, N.; Andrews-Pfannkoch, C.; Denisova, E. A.; Young, L.; Qi, Z.-Q.; Segall-Shapiro, T. H.; Calvey, C. H.; Parmar, P. P.; Hutchison, C. A.; Smith, H. O.; Venter, J. C. Creation of a Bacterial Cell 
Controlled by a Chemically Synthesized Genome. Science 2010, 329 (5987), 52-56.

(69) Annaluru, N.; Muller, H.; Mitchell, L. A.; Ramalingam, S.; Stracquadanio, G.; Richardson, S. M.; Dymond, J. S.; Kuang, Z.; Scheifele, L. Z.; Cooper, E. M.; Cai, Y.; Zeller, K.; Agmon, N.; Han, J. S.; Hadjithomas, M.; Tullman, J.; Caravelli, K.; Cirelli, K.; Guo, Z.; London, V.; Yeluru, A.; Murugan, S.; Kandavelou, K.; Agier, N.; Fischer, G.; Yang, K.; Martin, J. A.; Bilgel, M.; Bohutskyi, P.; Boulier, K. M.; Capaldo, B. J.; Chang, J.; Charoen, K.; Choi, W. J.; Deng, P.; DiCarlo, J. E.; Doong, J.; Dunn, J.; Feinberg, J. I.; Fernandez, C.; Floria, C. E.; Gladowski, D.; Hadidi, P.; Ishizuka, I.; Jabbari, J.; Lau, C. Y. L.; Lee, P. A.; Li, S.; Lin, D.; Linder, M. E.; Ling, J.; Liu, J.; Liu, J.; London, M.; Ma, H.; Mao, J.; McDade, J. E.; McMillan, A.; Moore, A. M.; Oh, W. C.; Ouyang, Y.; Patel, R.; Paul, M.; Paulsen, L. C.; Qiu, J.; Rhee, A.; Rubashkin, M. G.; Soh, I. Y.; Sotuyo, N. E.; Srinivas, V.; Suarez, A.; Wong, A.; Wong, R.; Xie, W. R.; Xu, Y.; Yu, A. T.; Koszul, R.; Bader, J. S.; Boeke, J. D.; Chandrasegaran, S. Total Synthesis of a Functional Designer Eukaryotic Chromosome. Science 2014, 344 (6179), 55-58.

(70) Garcia-Morales, L.; Ruiz, E.; Gourgues, G.; Rideau, F.; PiñeroLambea, C.; Lluch-Senar, M.; Blanchard, A.; Lartigue, C. A RAGE Based Strategy for the Genome Engineering of the Human Respiratory Pathogen Mycoplasma pneumoniae. ACS Synth. Biol. 2020, 9 (10), 2737-2748.

(71) Calero, P.; Volke, D. C.; Lowe, P. T.; Gotfredsen, C. H.; O’Hagan, D.; Nikel, P. I. A Fluoride-Responsive Genetic Circuit Enables in Vivo Biofluorination in Engineered Pseudomonas putida. Nat. Commun. 2020, 11 (1), 5045.

(72) Asin-Garcia, E.; Kallergi, A.; Landeweerd, L.; Martins dos Santos, V. A. P. Genetic Safeguards for Safety-by-Design: So Close Yet So Far. Trends Biotechnol. 2020, 38, 1308.

(73) Green, R.; Rogers, E. J. Transformation of Chemically Competent E. coli. In Methods in Enzymology; Elsevier, 2013; Vol. 529, pp 329-336. DOI: 10.1016/B978-0-12-418687-3.00028-8.

(74) Choi, K.-H.; Kumar, A.; Schweizer, H. P. A 10-min Method for Preparation of Highly Electrocompetent Pseudomonas aeruginosa Cells: Application for DNA Fragment Transfer between Chromosomes and Plasmid Transformation. J. Microbiol. Methods 2006, 64 (3), 391-397.

(75) Wirth, N. T.; Kozaeva, E.; Nikel, P. I. Accelerated Genome Engineering of Pseudomonas putida by I- Sce I-mediated Recombination and CRISPR -Cas9 Counterselection. Microb. Biotechnol. 2020, 13 (1), 233-249.

(76) Damalas, S. G.; Batianis, C.; Martin-Pascual, M.; Lorenzo, V. de; dos Santos, V. A. P. M. SEVA 3.1: Enabling Interoperability of DNA Assembly among the SEVA, BioBricks and Type IIS Restriction Enzyme Standards. Microb. Biotechnol. 2020, 13 (6), 1793-1806.

(77) Salis, H. M.; Mirsky, E. A.; Voigt, C. A. Automated Design of Synthetic Ribosome Binding Sites to Control Protein Expression. Nat. Biotechnol. 2009, 27 (10), 946-950.

(78) Batianis, C.; Kozaeva, E.; Damalas, S. G.; Martín-Pascual, M.; Volke, D. C.; Nikel, P. I.; Martins dos Santos, V. A. P. An Expanded CRISPRi Toolbox for Tunable Control of Gene Expression in Pseudomonas putida. Microb. Biotechnol. 2020, 13 (2), 368-385.

(79) Zuker, M. Mfold Web Server for Nucleic Acid Folding and Hybridization Prediction. Nucleic Acids Res. 2003, 31 (13), 34063415 .

(80) Aparicio, T.; Nyerges, A.; Nagy, I.; Pal, C.; Martínez-García, E.; Lorenzo, V. Mismatch Repair Hierarchy of Pseudomonas putida Revealed by Mutagenic SsDNA Recombineering of the PyrF Gene. Environ. Microbiol. 2020, 22 (1), 45-58. 